\title{
A COUNTER EXAMPLE TO CERCIGNANI'S CONJECTURE FOR THE D DIMENSIONAL KAC MODEL - AMIT EINAV
}

\author{
AMIT EINAV
}

\begin{abstract}
Aвstract. In this paper we extend results obtained in [6] to the $d$ dimensional Kac model, showing that an entropy-entropy production method in its full generality is unhelpful in finding an exponential rate of relaxation.
\end{abstract}

\section{INTRODUCTION}

In his 1956 paper [8], Marc Kac introduced a linear $N$-particle binary collision model from which, under the additional assumption of the newly defined 'Boltzmann Property' (what we now call chaotic families), a one dimensional caricature of the Boltzmann equation arose.

The the evolution equation for the one dimensional model (the 'master equation') was given by

$$
\frac{\partial F}{\partial t}\left(v_{1}, \ldots, v_{N}\right)=N(I-Q) F\left(v_{1}, \ldots, v_{N}\right)
$$

where

$$
Q F\left(v_{1}, \ldots, v_{N}\right)=\frac{1}{2 \pi} \cdot \frac{2}{N(N-1)} \sum_{i<j} \int_{0}^{2 \pi} F\left(v_{1}, \ldots, v_{i}(\vartheta), \ldots, v_{j}(\vartheta), \ldots, v_{N}\right) d \vartheta
$$

with

$$
\begin{gathered}
v_{i}(\vartheta)=v_{i} \cos (\vartheta)+v_{j} \sin (\vartheta) \\
v_{j}(\vartheta)=-v_{i} \sin (\vartheta)+v_{j} \cos (\vartheta)
\end{gathered}
$$

While the model itself wasn't completely physical, as it doesn't conserve momentum, it still gave rise to many interesting observations and results. One such observation was that the evolution equation (1.1) is ergodic on $\mathbb{S}^{N-1}(\sqrt{N})$ and $\lim _{t \rightarrow \infty} F\left(t, v_{1}, \ldots, v_{n}\right)=1$ for any fixed $N$. This led Kac to hope that a rate of relaxation of his linear equation can be bounded independently of $N$ and serve to prove a rate of relaxation in the associated Boltzmann equation. This so called 'spectral gap' problem was investigated by many people including Janvresse ([7]) and Maslen ([9]), and was finally given an explicit answer by Carlen, Carvahlo and Loss ([2]).

The fact that solving the above problem would not lead to a good enough relaxation rate was well known before the problem was solved. The $L^{2}$ norm, which is the source of the spectral gap evaluation, is catastrophic for chaotic families. A new, better, 'norm'-like function was required.

The author was supported by EPSRC grant MATKIT. 
A natural quantity to investigate, one that is much more amiable towards chaotic families, is the entropy. It is defined as

$$
H_{N}(F)=\int_{\mathbb{S}^{N-1}(\sqrt{N})} F \log F d \sigma^{N}
$$

where $d \sigma^{N}$ is the uniform probability measure on the sphere.

A related 'spectral gap' problem appeared: Can we find $C>0$ such that the entropic 'spectral gap' under (1.1), $\Gamma_{N}$, defined as

$$
\Gamma_{N}=\inf _{F \in L_{s y m}^{2}\left(\mathbb{S}^{N-1}(\sqrt{N})\right)} \frac{\langle\log F, N(I-Q) F\rangle}{H_{N}(F)}
$$

satisfies $\Gamma_{N}>C$ ?

The answer to that is No. In 2003 Villani managed to prove that $\Gamma_{N} \geq \frac{2}{N-1}$ and conjectured that $\Gamma_{N}=O\left(\frac{1}{N}\right)$ (see [12]) and later on in 2011 we managed to show that for any $0<\eta<1$ there exists $C_{\eta}>0$ such that $\Gamma_{N} \leq \frac{C_{\eta}}{N^{\eta}}$ (see [6]).

While the one dimensional model itself posed, and still posses, many interesting problem, the fact that it is not very physical is a small deterrent. In his 1967 paper [10], McKean generalized Kac's Model to a more realistic, momentum and energy conserving, model possessing many of the properties of the one dimensional model.

The evolution equation to the $d$-dimensional model is given by

$$
\frac{\partial F}{\partial t}\left(v_{1}, \ldots, v_{N}\right)=N(I-Q) F\left(v_{1}, \ldots, v_{N}\right)
$$

where $v_{1}, \ldots, v_{N} \in \mathbb{R}^{d}$ and

$$
Q F\left(v_{1}, \ldots, v_{N}\right)=\frac{2}{N(N-1)} \sum_{i<j} \int_{\mathbb{S}^{d-1}} F\left(v_{1}, \ldots, v_{i}(\omega), \ldots, v_{j}(\omega), \ldots, v_{N}\right) d \sigma^{d}
$$

with

$$
\begin{aligned}
& v_{i}(\omega)=\frac{v_{i}+v_{j}}{2}+\frac{\left|v_{i}-v_{j}\right|}{2} \cdot \omega \\
& v_{j}(\omega)=\frac{v_{i}+v_{j}}{2}-\frac{\left|v_{i}-v_{j}\right|}{2} \cdot \omega
\end{aligned}
$$

The appropriate space is no longer the energy sphere $\mathbb{S}^{N-1}(\sqrt{N})$, but the Boltzmann sphere, defined by

$$
\mathscr{S}_{B}^{N}(E, z)=\left\{v_{1}, \ldots,\left.v_{N} \in \mathbb{R}^{d}\left|\sum_{i=1}^{N}\right| v_{i}\right|^{2}=E, \sum_{i=1}^{N} v_{i}=z\right\}
$$

with $E=N$ and $z=0$ for simplicity. For more information we refer the reader to [4].

The related spectral gap problem was solved in 2008 by Carlen, Geronimo and Loss (see [4]), but a similar reasoning to that presented in the one dimensional case leads us to conclude that the spectral gap method is not suited to deal with 
chaotic families.

Like before, we define the entropy on the Boltzmann sphere

$$
H_{N}(F)=\int_{\mathscr{S}_{B}^{N}(N, 0)} F \log F d \sigma_{N, 0}^{N}
$$

where $d \sigma_{E, z}^{N}$ is the uniform probability measure on the Boltzmann sphere. Similarly to the one dimensional case we can define the entropic spectral gap as in (1.2):

$$
\Gamma_{N}=\inf _{F \in L_{s y m}^{2}\left(\mathscr{C}_{B}^{N}(N, 0)\right)} \frac{\langle\log F, N(I-Q) F\rangle}{H_{N}(F)}
$$

The main goal of this paper is to extend the result proved in [6] from one dimension to $d$ dimension, showing that in its full generality, the above entropyentropy production ratio doesn't give a good relaxation rate.

The main theorem of this paper is

Theorem 1.1. For any $0<\eta<1$ there exists a constant $C_{\eta}$, depending only on $\eta$, such that $\Gamma_{N}$, defined in (1.5), satisfies

$$
\Gamma_{N} \leq \frac{C_{\eta}}{N^{\eta}}
$$

The idea behind this proof is one that keeps repeating (see [6, 3]). An intuitive way to create a chaotic family on the Boltzmann sphere is by tensorising a one variable function (what we call our 'generating function'):

$$
F_{N}\left(v_{1}, \ldots, v_{N}\right)=\frac{\prod_{i-1}^{N} f\left(v_{i}\right)}{\mathfrak{Z}_{N}(f, \sqrt{N}, 0)}
$$

where the normalization function $\mathfrak{Z}_{N}$ is defined by

$$
\mathcal{Z}_{N}(f, \sqrt{u}, z)=\int_{\mathscr{S}_{B}^{N}(u, z)} \prod_{i=1}^{N} f\left(v_{i}\right) d \sigma_{u, z}^{N}
$$

The case where $f$ is a fixed function, extending results from the one dimensional case presented in [3], can be found in recent work by Carraptoso (see [5]). However, in order to prove our main result we will need to allow the 'generating function' to change with $N$ as well, much like [6].

The above introduction is, by far, a mere glimpse into the Kac Model and its relation to the Boltzmann equation. There are many more details and some remarkable proofs involved with this subject and we refer the reader to [2, 3, 4, 11, 13] to read more about it.

The paper is structured as follows: Section 2 will discuss some preliminaries, giving more information about the Boltzmann sphere and the normalization function. Section 3 will contain our specific choice of 'generating function' and the approximation theorem of its normalization function, leading to Section 4 where we prove the main theorem. Section 5 concludes with final words and some remarks and is followed by the Appendix, containing additional computation we found unnecessary to include in the main body of the paper. 
Acknowledgement: The author would like to thank Dr. Clément Mouhot for many fruitful discussions and constant encouragement, as well as Kleber Carrapatoso for allowing him to read the preprint of his paper ([5]), helping to bridge the dimension gap.

\section{Preliminaries}

In this section we'll discuss a few preliminary results, mainly about the Boltzmann sphere and the normalization function $Z_{N}(f, \sqrt{u}, z)$. Most of the results presented here can be found in [5] but we choose to present a variant of them for completion.

2.1. The Boltzmann Sphere. As mentioned before, the Boltzmann sphere is defined as

$$
\mathscr{S}_{B}^{N}(E, z)=\left\{v_{1}, \ldots,\left.v_{N} \in \mathbb{R}^{d}\left|\sum_{i=1}^{N}\right| v_{i}\right|^{2}=E, \sum_{i=1}^{N} v_{i}=z\right\}
$$

The term 'Boltzmann sphere' is evident from the following 'transformation':

$$
U=R V
$$

where $V=\left(v_{1}, \ldots, v_{N}\right)^{T}$ and $R$ is the orthogonal matrix with rows given by

$$
\begin{gathered}
r_{j}=\frac{1}{\sqrt{j(j+1)}}\left(\sum_{i=1}^{j} e_{i}-j e_{j+1}\right) \quad 1 \leq j \leq N-1 \\
r_{N}=\frac{\sum_{i=1}^{N} e_{i}}{\sqrt{N}}
\end{gathered}
$$

where $e_{j} \in \mathbb{R}^{N}$ is the standard basis. Under (2.1) we see that

$$
\mathscr{S}_{B}^{N}(E, z)=\left\{u_{1}, \ldots,\left.u_{N} \in \mathbb{R}^{d}\left|\sum_{i=1}^{N-1}\right| u_{i}\right|^{2}=E-\frac{|z|^{2}}{N}, u_{N}=\frac{z}{\sqrt{N}}\right\}
$$

giving us a sphere in a hyperplane of $d(N-1)$ dimensions of $\mathbb{R}^{d N}$ with radius $\sqrt{E-\frac{|z|^{2}}{N}}$

Next, since we'll be interested in integration with respect to the uniform probability measure on the Boltzmann sphere, $d \sigma_{E, z}^{N}$, we will need the following Fubini-type formula:

Theorem 2.1.

$$
\begin{gathered}
\int_{\mathscr{S}_{B}^{N}(E, z)} F d \sigma_{E, z}^{N}=\frac{\left|\mathbb{S}^{d(N-j-1)-1}\right|}{\left|\mathbb{S}^{d(N-1)-1}\right|} \cdot \frac{N^{\frac{d}{2}}}{(N-j)^{\frac{d}{2}}\left(E-\frac{|z|^{2}}{N}\right)^{\frac{d(N-1)-2}{2}}} \\
\int_{\Pi_{j}(E, z)} d v_{1} \ldots d v_{j}\left(E-\sum_{i=1}^{j}\left|v_{i}\right|^{2}-\frac{\left|z-\sum_{i=1}^{j} v_{i}\right|^{2}}{N-j}\right)^{\frac{d(N-j-1)-2}{2}} \\
\int_{\mathscr{S}_{B}^{N-j}\left(E-\sum_{i=i}^{j}\left|v_{i}\right|^{2}, z-\sum_{i=1}^{j} v_{i}\right)} F d \sigma_{E-\sum_{i=i}^{j}\left|v_{i}\right|^{2}, z-\sum_{i=1}^{j} v_{i}}^{N-j}
\end{gathered}
$$


where $\Pi_{j}(E, z)=\left\{\sum_{i=1}^{j}\left|v_{i}\right|^{2}+\frac{\left|z-\sum_{i=1}^{j} v_{i}\right|^{2}}{N-j} \leq E\right\}$.

We leave the proof to the Appendix (See Theorem A.1).

2.2. The Normalization Function. A key part of the proof of our main theorem lies with an approximation of the appropriate normalization function. While the true approximation theorem will be discussed in Section 3, we present here some basic probabilistic interpretation of it as a prelude to the proof.

As was mentioned before, the normalization function for a suitable function $f$ is defined as

$$
\mathfrak{Z}_{N}(f, \sqrt{r}, z)=\int_{\mathscr{S}_{B}^{N}(r, z)} f^{\otimes N} d \sigma_{r, z}^{N}
$$

Lemma 2.2. Let $V$ be a random variable with values in $\mathbb{R}^{d}$ and law $f$. Let $h$ be the law of the couple $\left(V,|V|^{2}\right)$ then

$$
Z_{N}(f, \sqrt{u}, z)=\frac{2 N^{\frac{d}{2}} h^{* N}(z, u)}{\left|\mathbb{S}^{d(N-1)-1}\right|\left(u-\frac{|z|^{2}}{N}\right)^{\frac{d(N-1)-2}{2}}}
$$

Proof. Let $\varphi \in C_{b}$ be a function of $\sum_{i=1}^{N} v_{i}$ and $\sum_{i=1}^{N}\left|v_{i}\right|^{2}$. By the definition

$$
\mathbb{E} \varphi=\int_{\mathbb{R}^{d N}} \varphi\left(\sum_{i=1}^{N} v_{i}, \sum_{i=1}^{N}\left|v_{i}\right|^{2}\right) f^{\otimes N}\left(v_{1}, \ldots, v_{N}\right) d v_{1} \ldots d v_{N}
$$

Using (2.1) we can rewrite the above as

$$
\begin{gathered}
\int_{\mathbb{R}^{d N}} \varphi\left(\sqrt{N} u_{N}, \sum_{i=1}^{N}\left|u_{i}\right|^{2}\right) f^{\otimes N} \circ R^{-1}\left(u_{1}, \ldots, u_{N}\right) d u_{1} \ldots d u_{N} \\
=\int_{\mathbb{R}^{d}} d u_{N} \int_{\mathbb{R}^{d(N-1)}} \varphi\left(\sqrt{N} u_{N}, \sum_{i=1}^{N}\left|u_{i}\right|^{2}\right) f^{\otimes N} \circ R^{-1}\left(u_{1}, \ldots, u_{N}\right) d u_{1} \ldots d u_{N} \\
=\int_{\mathbb{R}^{d}} d u_{N} \int_{0}^{\infty} d r \cdot r^{d(N-1)-1} \varphi\left(\sqrt{N} u_{N}, r^{2}+\left|u_{N}\right|^{2}\right) \int_{\mathbb{S}^{d(N-1)-1}} f^{\otimes N} \circ R^{-1} d s^{d(N-1)} \\
=\int_{\mathbb{R}^{d}} d u_{N} \int_{0}^{\infty} d r\left|\mathbb{S}^{d(N-1)-1}\right| r^{d(N-1)-1} \varphi\left(\sqrt{N} u_{N}, r^{2}+\left|u_{N}\right|^{2}\right) \int_{\mathbb{S}^{d(N-1)-1}} f^{\otimes N} \circ R^{-1} d \gamma^{d(N-1)}
\end{gathered}
$$

where $d \gamma$ is the uniform probability measure on the sphere.

At this point we notice that

$$
\begin{aligned}
& \int_{\mathbb{S} d(N-1)-1} f^{\otimes N} \circ R^{-1} d \gamma^{d(N-1)}=\int_{\sum_{i=1}^{N-1}\left|u_{i}\right|^{2}=r^{2}, u_{N}} f^{\otimes N} \circ R^{-1} d \gamma^{d(N-1)} \\
= & \int_{\sum_{i=1}^{N}\left|v_{i}\right|^{2}=r^{2}+\left|u_{N}\right|^{2}, \sum_{i=1}^{N} v_{i}=\sqrt{N} u_{N}} f^{\otimes N} d \sigma^{N}=Z_{N}\left(f, \sqrt{r^{2}+\left|u_{N}\right|^{2}}, \sqrt{N} u_{N}\right)
\end{aligned}
$$

Using the change of variables $z=\sqrt{N} u_{N}$ and $w=r^{2}+\left|u_{N}\right|^{2}$ yields

$\mathbb{E} \varphi=\int_{\mathbb{R}^{d}} d u_{N} \int_{0}^{\infty} d r\left|\mathbb{S}^{d(N-1)-1}\right|\left(r^{2}\right)^{\frac{d(N-1)-1}{2}} \varphi\left(\sqrt{N} u_{N}, r^{2}+\left|u_{N}\right|^{2}\right) \cdot Z_{N}\left(f, \sqrt{r^{2}+\left|u_{N}\right|^{2}}, \sqrt{N} u_{N}\right)$ 


$$
\begin{gathered}
=\int_{\mathbb{R}^{d}} d u_{N} \int_{0}^{\infty} d w \frac{\left|\mathbb{S}^{d(N-1)-1}\right|}{2}\left(w-\left|u_{N}\right|^{2}\right)^{\frac{d(N-1)-2}{2}} \varphi\left(\sqrt{N} u_{N}, w\right) \cdot \mathcal{Z}_{N}\left(f, \sqrt{w}, \sqrt{N} u_{N}\right) \\
\int_{\mathbb{R}^{d}} d z \int_{0}^{\infty} d w \frac{\left|\mathbb{S}^{d(N-1)-1}\right|}{2 N^{\frac{d}{2}}}\left(w-\frac{|z|^{2}}{N}\right)^{\frac{d(N-1)-2}{2}} \varphi(z, w) \cdot \mathcal{Z}_{N}(f, \sqrt{w}, z)
\end{gathered}
$$

On the other hand, denoting by $s_{N}$ the law of the couple $\sum_{i=1}^{N}\left(V_{i},\left|V_{i}\right|^{2}\right)=\left(\sum_{i=1}^{N} V_{i},\left.\sum_{i=1}^{n}|| V_{i}\right|^{2}\right)$ we find that

$$
\mathbb{E} \varphi=\int_{\mathbb{R}^{d} \times[0, \infty)} \varphi(z, w) s_{N}(z, w) d z d w
$$

This leads to the conclusion that

$$
Z_{N}(f, \sqrt{w}, z)=\frac{2 N^{\frac{d}{2}} s_{N}(z, w)}{\left|\mathbb{S}^{d(N-1)-1}\right|\left(w-\frac{|z|^{2}}{N}\right)^{\frac{d(N-1)-2}{2}}}
$$

and the result follows using a known theorem in Probability Theory.

The fact that convolution itself gives us a function and not just a law is discussed in [5]. In our particular case we'll prove that we indeed get a well defined function upon a very specific choice of law $f$.

We conclude this section with the connection between the law of $V$ and the couple $\left(V,|V|^{2}\right)$.

Lemma 2.3. Let $f$ be a density function for the random variable $V$. Then, the law of the couple $\left(V,|V|^{2}\right)$, denoted by $h$, is given by

$$
d h(v, u)=f(v) \delta_{u=|v|^{2}}(u) d v d u
$$

Proof. let $\varphi \in C_{b}$ be a function of $\nu$. Then

$$
\mathbb{E} \varphi=\int_{\mathbb{R}^{d}} \varphi(v) f(v) d v
$$

on the other hand

$$
\mathbb{E} \varphi=\int_{\mathbb{R}^{d} \times[0, \infty)} \varphi(v) h(v, u) d v d u
$$

Since every function of the couple $\left(v,|v|^{2}\right)$ is actually a function of $v$. The result follows.

\section{The Normalization Function AND its Approximation}

The core of the proof presented in [6] lies in understanding how the normalization function of a particular family of densities behaves asymptotically on the Kac sphere. The same understanding is the crucial step in proving our main result.

Unlike [6], where we proved a general approximation type theorem, our current situation is more complex and technical, and as such we restrict ourselves to proving the approximation theorem for the specific choice of 'generating function'.

The first step we must take is to define the 'generating function'. This is a very 
natural choice following the trends of $[1,3,6]$.

Definition 3.1. We denote by

$$
f_{\delta}(v)=\delta M_{\frac{1}{2 d \delta}}(\nu)+(1-\delta) M_{\frac{1}{2 d(1-\delta)}}(v)
$$

where $M_{a}(v)=\frac{1}{(2 \pi a)^{\frac{d}{2}}} e^{-\frac{|\nu|^{2}}{2 a}}$.

Lemma 3.2. Let $f_{\delta}$ be as defined in (3.1). Then

$$
\widehat{h_{\delta}}(p, t)=\frac{\delta e^{-\frac{\pi^{2}|p|^{2}}{d \delta+2 \pi i t}}}{\left(1+\frac{2 \pi i t}{d \delta}\right)^{\frac{d}{2}}}+\frac{(1-\delta) e^{-\frac{\pi^{2}|p|^{2}}{d(1-\delta)+2 \pi i t}}}{\left(1+\frac{2 \pi i t}{d(1-\delta)}\right)^{\frac{d}{2}}}
$$

where $h_{\delta}$ is associated to $f_{\delta}$ via Lemma 2.3 and the Fourier transform is defined in the measure sense.

Proof. We begin with the known Fourier transform of the Gaussian

$$
\int_{\mathbb{R}} e^{-\beta x^{2}} e^{-2 \pi i x \xi} d x=\sqrt{\frac{\pi}{\beta}} e^{-\frac{\pi^{2} \xi^{2}}{\beta}}
$$

for $\beta>0$. Since both sides are clearly analytic in $\beta$ for $\operatorname{Re} \beta>0$ we find that the equality is still true in that domain.

Denoting by $h_{a}$ the law associated to the couple $\left(V,|V|^{2}\right)$ where $V$ has law $M_{a}$, we notice that by the above remark, Lemma 2.3 and the definition of the Fourier transform of a measure:

$$
\begin{gathered}
\widehat{h_{a}}(p, t)=\int_{\mathbb{R}^{d} \times[0, \infty)} e^{-2 \pi i(p \circ v+t u)} d h(v, u)=\int_{\mathbb{R}^{d}} M_{a}(\nu) e^{-2 \pi i\left(p \circ \nu+t|v|^{2}\right)} \\
=\prod_{i=1}^{d} \frac{1}{\sqrt{2 \pi a}} \int_{\mathbb{R}} e^{-v_{i}^{2}\left(\frac{1}{2 a}+2 \pi i t\right)} \cdot e^{-2 \pi i p_{i} v_{i}} d v_{i}=\prod_{i=1}^{d} \frac{e^{-\frac{\pi^{2} p_{i}^{2}}{\frac{1}{2 a}+2 \pi i t}}}{\sqrt{1+4 \pi i a t}}=\frac{e^{-\frac{2 a \pi^{2}|p|^{2}}{1+4 \pi i a t}}}{(1+4 \pi i a t)^{\frac{d}{2}}}
\end{gathered}
$$

thus the result follows immediately from the definition of $f_{\delta}$ and the linearity of the Fourier transform.

At this point we'll explain why the convolution in (2.4) yields a function. The proof of the Lemma is provided in the Appendix.

Lemma 3.3. Let $h_{\delta}$ be associated to $f_{\delta}$ via Lemma 2.3 where $f_{\delta}$ is defined in (3.1). Then ${\widehat{h_{\delta}}}^{n} \in L^{q}\left(\mathbb{R}^{d} \times[0, \infty)\right)$ when $n>\frac{2(1+d)}{q d}$. In particular, for every $n>\frac{2(1+d)}{d}$ we have that ${\widehat{h_{\delta}}}^{n} \in L^{2}\left(\mathbb{R}^{d} \times[0, \infty)\right) \cap L^{1}\left(\mathbb{R}^{d} \times[0, \infty)\right)$ and thus $h^{* n}$ can be viewed as a density.

We are now ready to state the main theorem of this section: 
Theorem 3.4. Let $f_{\delta_{N}}$ be as in (3.1) where $\delta_{N}=\frac{1}{N^{1-\eta}}$ with $\frac{2 \beta}{1+2 \beta}<\eta<\frac{(3+d) \beta}{1+3 \beta+\frac{d}{2}+d \beta}$ and $0<\beta<1$ arbitrary. Then

$$
\sup _{u \in[0, \infty), v \in \mathbb{R}^{d}}\left|\frac{\left|\mathbb{S}^{d(N-1)-1}\right|\left(u-\frac{|z|^{2}}{N}\right)^{\frac{d(N-1)-2}{2}}}{2 N^{\frac{d}{2}}} Z_{N}\left(f_{\delta_{N}}, \sqrt{u}, v\right)-\gamma_{N}(u, v)\right| \leq \frac{\epsilon(N)}{\sum_{\delta_{N}}^{2} N^{\frac{d+1}{2}}}
$$

where $\gamma_{N}(u, v)=\frac{d^{\frac{d}{2}}}{\Sigma_{\delta_{N}}^{2} N^{\frac{d+1}{2}}} \cdot \frac{e^{-\frac{d|\nu|^{2}}{2 N}} \cdot e^{-\frac{(u-N)^{2}}{2 \Sigma_{\delta_{N}}^{2} N}}}{(2 \pi)^{\frac{d+1}{2}}}$ and $\Sigma_{\delta_{N}}^{2}=\frac{d+2}{4 d \delta_{N}\left(1-\delta_{N}\right)}-1$

Remark 3.5. The above approximation theorem gives a similar result to the one presented in [5], however a closer inspection of our choice of 'generating function' shows a difference in the definition of $\Sigma$. We believe this difference manifests itself due to the dependency of $\delta$ in $N$, appearing as a different dimension factor.

The proof of the above theorem is quite technical and requires a division of $\mathbb{R}^{d} \times \mathbb{R}$ into several domains. In order to do it in an orderly fashion we will prove integral estimations of the Fourier transform of the mentioned function in each regime in several subsections. We would encourage the reader to skip the rest of this section and jump to Section 4 to see how the approximation theorem serves to prove the main result.

The phasespace domain $(p, t) \in \mathbb{R}^{d} \times \mathbb{R}$ will be divided into
i. $|t|>\frac{d \delta}{4 \pi}, p \in \mathbb{R}^{d}$.
ii. $|t| \leq \frac{d \delta}{4 \pi},|p|>\eta$.
iii. $\frac{d \delta^{1+\beta}}{4 \pi}<|t| \leq \frac{d \delta}{4 \pi},|p| \leq \eta$.
iv. $|t|<\frac{d \delta^{1+\beta}}{4 \pi},|p| \leq \eta$

where in the last step we'll choose $\eta=\delta^{\frac{1}{2}+\beta}$.

Before we begin with each domain we'd like to state a couple of Lemmas.

Lemma 3.6. For any $\alpha, \beta>0$ we have that

$$
\begin{array}{r}
\int_{x>\beta} e^{-\alpha x^{2}} d x \leq \sqrt{\frac{\pi}{4 \alpha}} e^{-\frac{\alpha \beta^{2}}{2}} \\
\int_{x>\beta} x e^{-\alpha x^{2}} d x \leq \frac{e^{-\frac{\alpha \beta^{2}}{2}}}{2 \alpha} \\
\int_{0<x \leq \beta} e^{-\alpha x^{2}} d x \leq \sqrt{\frac{\pi}{4 \alpha}} \sqrt{1-e^{-2 \alpha \beta^{2}}}
\end{array}
$$

Proof. This follows immediately from the next estimations

$$
\int_{x>\beta} e^{-\alpha x^{2}} d x=\frac{1}{\sqrt{\alpha}} \int_{x>\sqrt{\alpha} \beta} e^{-x^{2}} d x=\frac{1}{\sqrt{\alpha}} \sqrt{\int_{x, y>\sqrt{\alpha} \beta} e^{-x^{2}-y^{2}} d x d y}
$$


A COUNTER EXAMPLE TO CERCIGNANI'S CONJECTURE FOR THE D DIMENSIONAL KAC MODEL - AMIT EINAY

$$
\begin{gathered}
\leq \frac{1}{\sqrt{\alpha}} \sqrt{\int_{x^{2}+y^{2}>\alpha \beta^{2}, x>0, y>0} e^{-x^{2}-y^{2}} d x d y}=\sqrt{\frac{\pi}{2 \alpha}} \sqrt{\int_{r>\sqrt{\alpha} \beta} r e^{-r^{2}} d r}=\sqrt{\frac{\pi}{4 \alpha}} e^{-\frac{\alpha \beta^{2}}{2}} \\
\int_{x>\beta} x e^{-\alpha x^{2}} d x=\frac{1}{\alpha} \int_{x>\sqrt{\alpha} \beta} x e^{-x^{2}} d x=\frac{e^{-\alpha \beta^{2}}}{2 \alpha} \leq \frac{e^{-\frac{\alpha \beta^{2}}{2}}}{2 \alpha} \\
\int_{x \leq \beta} e^{-\alpha x^{2}} d x \leq \frac{1}{\sqrt{\alpha}} \sqrt{\int_{x^{2}+y^{2} \leq 2 \alpha \beta^{2}, x>0, y>0} e^{-x^{2}-y^{2}} d x d y}=\sqrt{\frac{\pi}{2 \alpha}} \sqrt{\int_{r<\sqrt{2 \alpha} \beta} r e^{-r^{2}} d r} \\
=\sqrt{\frac{\pi}{4 \alpha}} \sqrt{1-e^{-2 \alpha \beta^{2}}}
\end{gathered}
$$

\section{Lemma 3.7.}

$$
\int_{|x|>\beta}|x|^{m} e^{-\alpha|x|^{2}} d^{d} x \leq \frac{C_{m, d} \max \left(\beta^{m+d-2}, \beta^{m+d-4}, \ldots, 1\right)}{\min \left(\alpha, \alpha^{2}, \ldots, \alpha^{\left[\frac{m+d+2}{2}\right]}\right)} e^{-\frac{\alpha \beta^{2}}{2}}
$$

where $C_{m . d}$ is a constant depending only on $m$ and $d$.

Proof. First we notice that

$$
\int_{|x|>\beta}|x|^{m} e^{-\alpha|x|^{2}} d^{d} x=C_{d, m} \int_{r>\beta} r^{m+d-1} e^{-\alpha r^{2}} d r
$$

where $C_{d, m}$ is a constant depending only on $m$ and $d$.

Lemma 3.6 tells us that

$$
\int_{x>\beta} x^{j} e^{-\alpha x^{2}} d x \leq \frac{C e^{-\frac{\alpha \beta^{2}}{2}}}{\min (\alpha, \sqrt{\alpha})}
$$

where $j=0,1$. For $j>1$ we have that

$$
\begin{gathered}
\int_{x>\beta} x^{j} e^{-\alpha x^{2}} d x=\frac{1}{\alpha^{\frac{j+1}{2}}} \int_{x>\sqrt{\alpha} \beta} x^{j} e^{-x^{2}} d x \\
=\frac{1}{\alpha^{\frac{j+1}{2}}}\left(-\left.\frac{x^{j-1} e^{-x^{2}}}{2}\right|_{\sqrt{\alpha} \beta} ^{\infty}+\frac{j-1}{2} \int_{x>\sqrt{\alpha} \beta} x^{j-2} e^{-x^{2}} d x\right) \\
=\frac{1}{\alpha^{\frac{j+1}{2}}}\left(\frac{\alpha^{\frac{j-1}{2}} \beta^{j-1} e^{-\alpha \beta^{2}}}{2}+\frac{j-1}{2} \int_{x>\sqrt{\alpha} \beta} x^{j-2} e^{-x^{2}} d x\right)
\end{gathered}
$$

Continuing to integrate by parts yields

$$
\begin{gathered}
\int_{x>\beta} x^{j} e^{-\alpha x^{2}} d x \leq \frac{C_{j}}{\alpha^{\frac{j+1}{2}}}\left(\alpha^{\frac{j-1}{2}} \beta^{j-1} e^{-\alpha \beta^{2}}+\alpha^{\frac{j-3}{2}} \beta^{j-3} e^{-\alpha \beta^{2}}+\cdots+\int_{x>\sqrt{\alpha} \beta} x^{\tilde{j}} e^{-x^{2}} d x\right) \\
=C_{j}\left(\frac{\beta^{j-1} e^{-\alpha \beta^{2}}}{\alpha}+\frac{\beta^{j-3} e^{-\alpha \beta^{2}}}{\alpha^{2}}+\cdots+\frac{1}{\alpha^{\frac{j+1}{2}}} \int_{x>\sqrt{\alpha} \beta} x^{\tilde{j}} e^{-x^{2}} d x\right)
\end{gathered}
$$


where $C_{j}$ is a constant depending only on $j$ and $\tilde{j}=0,1$. Using our previous estimation we conclude that

$$
\int_{x>\beta} x^{j} e^{-\alpha x^{2}} d x \leq \frac{C_{j} \max \left(\beta^{j-1}, \beta^{j-3}, \ldots, 1\right)}{\min \left(\alpha, \alpha^{2}, \ldots, \alpha^{\left[\frac{j+3}{2}\right]}\right)} e^{-\frac{\alpha \beta^{2}}{2}}
$$

Thus, there exists a constant $C_{m, d}$, depending only on $m$ and $d$, such that

$$
\int_{|x|>\beta}|x|^{m} e^{-\alpha|x|^{2}} d^{d} x \leq \frac{C_{m, d} \max \left(\beta^{m+d-2}, \beta^{m+d-4}, \ldots, 1\right)}{\min \left(\alpha, \alpha^{2}, \ldots, \alpha^{\left[\frac{m+d+2}{2}\right]}\right)} e^{-\frac{\alpha \beta^{2}}{2}}
$$

Remark 3.8. In the special case where $\alpha \geq 1$ and $\beta \leq 1$ we get the estimation

$$
\int_{|x|>\beta}|x|^{m} e^{-\alpha|x|^{2}} d^{d} x \leq \frac{C_{m, d}}{\alpha} e^{-\frac{\alpha \beta^{2}}{2}}
$$

Lastly, before we start with the integral estimation, we notice three things:

(1) It is easy to show that $\widehat{\gamma}_{N}(p, t)={\widehat{\gamma_{1}}}^{N}(p, t)$ where

$$
\gamma_{1}(p, t)=e^{-\frac{2 \pi^{2}|p|^{2}}{d}} \cdot e^{-2 \pi i t} \cdot e^{-2 \pi^{2} \Sigma_{\delta}^{2} t^{2}}
$$

(2) An estimation we'll constantly use is the following: For any $0 \leq k \leq N-1$ we have that

$$
\begin{gathered}
|\widehat{h}(p, t)|^{k}\left|\widehat{\gamma_{1}}(p, t)\right|^{N-k-1} \\
\leq \sum_{j=0}^{k}\left(\begin{array}{c}
k \\
j
\end{array}\right) \frac{\delta^{j}}{\left(1+\frac{4 \pi^{2} t^{2}}{d^{2} \delta^{2}}\right)^{\frac{d j}{4}}} \frac{(1-\delta)^{k-j}}{\left(1+\frac{4 \pi^{2} t^{2}}{d^{2}(1-\delta)^{2}}\right)^{\frac{d(k-j)}{4}}} \\
\cdot e^{-\pi^{2}|p|^{2}\left(\frac{j d \delta}{d^{2} \delta^{2}+4 \pi^{2} t^{2}}+\frac{(k-j) d(1-\delta)}{d^{2}(1-\delta)^{2}+4 \pi^{2} t^{2}}+\frac{2(N-k-1)}{d}\right)} e^{-2 \pi^{2}(N-k-1) \Sigma^{2} t^{2}}
\end{gathered}
$$

(3) $|\widehat{h}(p, t)| \leq 1$ and $\left|\widehat{\gamma_{1}}(p, t)\right| \leq 1$.

3.1. Large $t$, any $p:|t|>\frac{d \delta}{4 \pi}$. The main theorem of this subsection is the following:

\section{Theorem 3.9.}

$$
\begin{gathered}
\iint_{\mathbb{R}^{d} \times|t|>\frac{d \delta}{4 \pi}}\left|\widehat{h}^{N}(p, t)-{\widehat{\gamma_{1}}}^{N}(p, t)\right| d p d t \leq \frac{N C_{d}}{(N-2)^{\frac{d+1}{2}} \Sigma} \cdot e^{-\frac{d(N-2) \Sigma^{2} \delta^{2}}{32}} \\
+\frac{N C_{d}}{2 \Sigma}\left(1-\left(1-\left(\frac{4}{5}\right)^{\frac{d}{4}}\right) \delta+\delta^{2} \varphi(\delta)\right)^{\frac{N}{2}} \cdot e^{-\frac{d^{2} \Sigma^{2} \delta^{2}}{32}}+C_{d}\left(1-\left(1-\left(\frac{4}{5}\right)^{\frac{d}{4}}\right) \delta+\delta^{2} \varphi(\delta)\right)^{N-5}
\end{gathered}
$$

where $C_{d}$ is a constant depending only ond and $\varphi$ is analytic in $|x|<\frac{1}{2}$. 
In order to prove the above theorem we need a series of Lemmas and small computations.

We start by noticing that due to (3.9) we have

$$
\begin{gathered}
\int_{\mathbb{R}^{d}}|\widehat{h}(p, t)|^{k}\left|\widehat{\gamma_{1}}(p, t)\right|^{N-k-1} d p \\
\leq C_{d} \sum_{j=0}^{k}\left(\begin{array}{c}
k \\
j
\end{array}\right) \frac{\delta^{j}}{\left(1+\frac{4 \pi^{2} t^{2}}{d^{2} \delta^{2}}\right)^{\frac{d j}{4}}} \frac{(1-\delta)^{k-j}}{\left(1+\frac{4 \pi^{2} t^{2}}{d^{2}(1-\delta)^{2}}\right)^{\frac{d(k-j)}{4}}} \cdot \frac{e^{-2 \pi^{2}(N-k-1) \Sigma^{2} t^{2}}}{\left(\frac{j d \delta}{d^{2} \delta^{2}+4 \pi^{2} t^{2}}+\frac{(k-j) d(1-\delta)}{d^{2}(1-\delta)^{2}+4 \pi^{2} t^{2}}+\frac{2(N-k-1)}{d}\right)^{\frac{d}{2}}}
\end{gathered}
$$

where $C_{d}=\frac{1}{\pi^{d}} \int_{\mathbb{R}^{d}} e^{-|z|^{2}} d z$.

Next, we see that

$$
\begin{gathered}
\frac{1}{\left(\frac{j d \delta}{d^{2} \delta^{2}+4 \pi^{2} t^{2}}+\frac{(k-j) d(1-\delta)}{d^{2}(1-\delta)^{2}+4 \pi^{2} t^{2}}+\frac{2(N-k-1)}{d}\right)^{\frac{d}{2}}} \\
\leq \min \left(\frac{\left(d^{2} \delta^{2}+4 \pi^{2} t^{2}\right)^{\frac{d}{2}}}{(j d \delta)^{\frac{d}{2}}}, \frac{\left(d^{2}(1-\delta)^{2}+4 \pi^{2} t^{2}\right)^{\frac{d}{2}}}{((k-j) d(1-\delta))^{\frac{d}{2}}}, \frac{d^{\frac{d}{2}}}{(2(N-k-1))^{\frac{d}{2}}}\right)
\end{gathered}
$$

In our domain we have that $4 \pi^{2} t^{2} \geq \frac{d^{2} \delta^{2}}{4}$ and so

$$
\begin{gathered}
d^{2} \delta^{2}+4 \pi^{2} t^{2} \leq 20 \pi^{2} t^{2} \\
d^{2}(1-\delta)^{2}+4 \pi^{2} t^{2} \leq d^{2}+20 \pi^{2} t^{2}
\end{gathered}
$$

leading to

$$
\max \left(\left(d^{2} \delta^{2}+4 \pi^{2} t^{2}\right)^{\frac{d}{2}},\left(d^{2}(1-\delta)^{2}+4 \pi^{2} t^{2}\right)^{\frac{d}{2}}\right) \leq A_{d}\left(1+|t|^{d}\right)
$$

where $A_{d}$ is a constant depending only on $d$. We are now ready to state and prove our first Lemma.

\section{Lemma 3.10.}

$$
\begin{gathered}
\sum_{k=0}^{\left[\frac{N}{2}\right]} \iint_{\mathbb{R}^{d} \times|t|>\frac{d \delta}{4 \pi}}\left|\widehat{h}(p, t)-\widehat{\gamma_{1}}(p, t)\right||\widehat{h}(p, t)|^{k}\left|\widehat{\gamma_{1}}(p, t)\right|^{N-k-1} d p d t \\
\leq \frac{N C_{d}}{(N-2)^{\frac{d+1}{2}} \Sigma} \cdot e^{-\frac{d(N-2) \Sigma^{2} \delta^{2}}{32}}
\end{gathered}
$$

where $C_{d}$ is a constant depending only on $d$.

Proof. Since $|\widehat{h}(p, t)| \leq 1$ and $\left|\widehat{\gamma_{1}}(p, t)\right| \leq 1$, we find that along with inequality (3.11), inequality (3.12) and the fact that $k \leq \frac{N}{2}$ we have

$$
\begin{gathered}
\sum_{k=0}^{\left[\frac{N}{2}\right]} \iint_{\mathbb{R}^{d} \times|t|>\frac{d \delta}{4 \pi}}\left|\widehat{h}(p, t)-\widehat{\gamma_{1}}(p, t)\right||\widehat{h}(p, t)|^{k}\left|\widehat{\gamma_{1}}(p, t)\right|^{N-k-1} d p d t \\
\leq \frac{2 C_{d} d^{\frac{d}{2}}}{(N-2)^{\frac{d}{2}}} \sum_{k=0}^{\left[\frac{N}{2}\right]} \int_{|t|>\frac{d \delta}{4 \pi}} \sum_{j=0}^{k}\left(\begin{array}{c}
k \\
j
\end{array}\right) \frac{\delta^{j}}{\left(1+\frac{4 \pi^{2} t^{2}}{d^{2} \delta^{2}}\right)^{\frac{d j}{4}}} \frac{(1-\delta)^{k-j}}{\left(1+\frac{4 \pi^{2} t^{2}}{d^{2}(1-\delta)^{2}}\right)^{\frac{d(k-j)}{4}} \cdot e^{-\pi^{2}(N-2) \Sigma^{2} t^{2}}}
\end{gathered}
$$




$$
\begin{gathered}
=\frac{2 C_{d} d^{\frac{d}{2}}}{(N-2)^{\frac{d}{2}}} \sum_{k=0}^{\left[\frac{N}{2}\right]} \int_{|t|>\frac{d \delta}{4 \pi}}\left(\frac{\delta}{\left(1+\frac{4 \pi^{2} t^{2}}{d^{2} \delta^{2}}\right)^{\frac{d}{4}}}+\frac{(1-\delta)}{\left(1+\frac{4 \pi^{2} t^{2}}{d^{2}(1-\delta)^{2}}\right)^{\frac{d}{4}}}\right)^{k} \cdot e^{-\pi^{2}(N-2) \Sigma^{2} t^{2}} \\
\leq \frac{2 C_{d} d^{\frac{d}{2}}}{(N-2)^{\frac{d}{2}}} \sum_{k=0}^{\left[\frac{N}{2}\right]} \int_{|t|>\frac{d \delta}{4 \pi}} e^{-\pi^{2}(N-2) \Sigma^{2} t^{2}} \leq \frac{N C_{d} d^{\frac{d}{2}}}{(N-2)^{\frac{d}{2}}} \int_{|t|>\frac{d \delta}{4 \pi}} e^{-\pi^{2}(N-2) \Sigma^{2} t^{2}} \\
\leq \frac{N C_{d}}{(N-2)^{\frac{d+1}{2}} \Sigma} \cdot e^{-\frac{d(N-2) \Sigma^{2} \delta^{2}}{32}}
\end{gathered}
$$

where the last inequality is due to Lemma 3.6. This concludes the proof.

In order to be able and continue we notice the following simple observation: For $|t|>\frac{d \delta}{4 \pi}$ we have that

$$
\frac{\delta}{\left(1+\frac{4 \pi^{2} t^{2}}{d^{2} \delta^{2}}\right)^{\frac{d}{4}}}+\frac{(1-\delta)}{\left(1+\frac{4 \pi^{2} t^{2}}{d^{2}(1-\delta)^{2}}\right)^{\frac{d}{4}}} \leq \frac{\delta}{\left(\frac{5}{4}\right)^{\frac{d}{4}}}+\frac{1-\delta}{\left(1+\frac{5 \delta^{2}}{4(1-\delta)^{2}}\right)^{\frac{d}{4}}}
$$

When $0<\delta<\frac{1}{2}$ we can expand the second expression as a power series

$$
\frac{1-\delta}{\left(1+\frac{5 \delta^{2}}{4(1-\delta)^{2}}\right)^{\frac{d}{4}}}=(1-\delta)\left(1-\frac{5 d \delta^{2}}{16(1-\delta)^{2}}+\ldots\right)=1-\delta+\delta^{2} \varphi(\delta)
$$

where $\varphi$ is analytic in $|x|<\frac{1}{2}$.

From the above we conclude that

$$
\frac{\delta}{\left(1+\frac{4 \pi^{2} t^{2}}{d^{2} \delta^{2}}\right)^{\frac{d}{4}}}+\frac{(1-\delta)}{\left(1+\frac{4 \pi^{2} t^{2}}{d^{2}(1-\delta)^{2}}\right)^{\frac{d}{4}}} \leq\left(1-\left(1-\left(\frac{4}{5}\right)^{\frac{d}{4}}\right) \delta+\delta^{2} \varphi(\delta)\right)
$$

We're now ready to state and prove our second Lemma.

\section{Lemma 3.11.}

$$
\begin{gathered}
\sum_{k=\left[\frac{N}{2}\right]+1}^{N-2} \iint_{\mathbb{R}^{d} \times|t|>\frac{d \delta}{4 \pi}}\left|\widehat{h}(p, t)-\widehat{\gamma_{1}}(p, t)\right||\widehat{h}(p, t)|^{k}\left|\widehat{\gamma_{1}}(p, t)\right|^{N-k-1} d p d t \\
\leq \frac{N C_{d}}{2 \Sigma}\left(1-\left(1-\left(\frac{4}{5}\right)^{\frac{d}{4}}\right) \delta+\delta^{2} \varphi(\delta)\right)^{\frac{N}{2}} \cdot e^{-\frac{d^{2} \Sigma^{2} \delta^{2}}{32}}
\end{gathered}
$$

where $C_{d}$ is a constant depending only ond and $\varphi$ is analytic in $|x|<\frac{1}{2}$.

Proof. Like in the proof of Lemma 3.10 we'll be using inequalities (3.11), (3.12) and the fact that $N-k-1 \geq 1$ to conclude that

$$
\begin{gathered}
\sum_{k=\left[\frac{N}{2}\right]+1}^{N-2} \iint_{\mathbb{R}^{d} \times|t|>\frac{d \delta}{4 \pi}}\left|\widehat{h}(p, t)-\widehat{\gamma_{1}}(p, t)\right||\widehat{h}(p, t)|^{k}\left|\widehat{\gamma_{1}}(p, t)\right|^{N-k-1} d p d t \\
\leq \frac{2 C_{d} d^{\frac{d}{2}}}{2^{\frac{d}{2}}} \sum_{k=\left[\frac{N}{2}\right]+1}^{N-2} \int_{|t|>\frac{d \delta}{4 \pi}} \sum_{j=0}^{k}\left(\begin{array}{c}
k \\
j
\end{array}\right) \frac{\delta^{j}}{\left(1+\frac{4 \pi^{2} t^{2}}{d^{2} \delta^{2}}\right)^{\frac{d j}{4}}} \frac{(1-\delta)^{k-j}}{\left(1+\frac{4 \pi^{2} t^{2}}{d^{2}(1-\delta)^{2}}\right)^{\frac{d(k-j)}{4}} \cdot e^{-\pi^{2} \Sigma^{2} t^{2}}}
\end{gathered}
$$


A COUNTER EXAMPLE TO CERCIGNANI'S CONJECTURE FOR THE D DIMENSIONAL KAC MODEL - AMIT EINAY

$$
\begin{gathered}
=C_{d} \sum_{k=\left[\frac{N}{2}\right]+1}^{N-2} \int_{|t|>\frac{d \delta}{4 \pi}}\left(\frac{\delta}{\left(1+\frac{4 \pi^{2} t^{2}}{d^{2} \delta^{2}}\right)^{\frac{d}{4}}}+\frac{(1-\delta)}{\left(1+\frac{4 \pi^{2} t^{2}}{d^{2}(1-\delta)^{2}}\right)^{\frac{d}{4}}}\right)^{k} \cdot e^{-\pi^{2} \Sigma^{2} t^{2}} \\
\leq \frac{N C_{d}}{2}\left(1-\left(1-\left(\frac{4}{5}\right)^{\frac{d}{4}}\right) \delta+\delta^{2} \varphi(\delta)\right)^{\frac{N}{2}} \int_{|t|>\frac{d \delta}{4 \pi}} e^{-\pi^{2} \Sigma^{2} t^{2}}
\end{gathered}
$$

and Lemma 3.6 yields the final estimation.

Lastly, we have the following Lemma:

\section{Lemma 3.12.}

$\iint_{\mathbb{R}^{d} \times|t|>\frac{d \delta}{4 \pi}}\left|\widehat{h}(p, t)-\widehat{\gamma_{1}}(p, t)\right||\widehat{h}(p, t)|^{N-1} d p d t \leq C_{d}\left(1-\left(1-\left(\frac{4}{5}\right)^{\frac{d}{4}}\right) \delta+\delta^{2} \varphi(\delta)\right)^{N-5}$

where $C_{d}$ is a constant depending only ond and $\varphi$ is analytic in $|x|<\frac{1}{2}$.

Proof. Using inequality (3.11), (3.12) and (3.13) with $k=N-1$ we find that

$$
\begin{gathered}
\iint_{\mathbb{R}^{d} \times|t|>\frac{d \delta}{4 \pi}}\left|\widehat{h}(p, t)-\widehat{\gamma_{1}}(p, t)\right||\widehat{h}(p, t)|^{N-1} d p d t \\
\leq C_{d} \int_{|t|>\frac{d \delta}{4 \pi}} \sum_{j=0}^{N-1}\left(\begin{array}{c}
N-1 \\
j
\end{array}\right) \frac{\delta^{j}}{\left(1+\frac{4 \pi^{2} t^{2}}{d^{2} \delta^{2}}\right)^{\frac{d j}{4}}} \frac{(1-\delta)^{N-1-j}}{\left(1+\frac{4 \pi^{2} t^{2}}{d^{2}(1-\delta)^{2}}\right)^{\frac{d(N-1-j)}{4}}} \\
\cdot \min \left(\frac{\left(1+|t|^{d}\right)}{(j d \delta)^{\frac{d}{2}}}, \frac{\left(1+|t|^{d}\right)}{((N-1-j) d(1-\delta))^{\frac{d}{2}}}\right) d t
\end{gathered}
$$

For $\delta<\frac{1}{2}$ and $0 \leq j \leq N-1$ we find that

$$
\min \left(\frac{\left(1+|t|^{d}\right)}{(j d \delta)^{\frac{d}{2}}}, \frac{\left(1+|t|^{d}\right)}{((N-1-j) d(1-\delta))^{\frac{d}{2}}}\right) \leq \frac{C_{d}\left(1+|t|^{d}\right)}{\left(\frac{\delta(N-1)}{2}\right)^{\frac{d}{2}}}
$$

Thus, our desired expression is bounded above by

$$
\begin{gathered}
\frac{C_{d}}{(\delta(N-1))^{\frac{d}{2}}} \int_{|t|>\frac{d \delta}{4 \pi}}\left(\frac{\delta}{\left(1+\frac{4 \pi^{2} t^{2}}{d^{2} \delta^{2}}\right)^{\frac{d}{4}}}+\frac{(1-\delta)}{\left(1+\frac{4 \pi^{2} t^{2}}{d^{2}(1-\delta)^{2}}\right)^{\frac{d}{4}}}\right)^{N-1}\left(1+|t|^{d}\right) d t \\
\leq \frac{C_{d}}{(\delta(N-1))^{\frac{d}{2}}}\left(1-\left(1-\left(\frac{4}{5}\right)^{\frac{d}{4}}\right) \delta+\delta^{2} \varphi(\delta)\right)^{N-5} \\
\int_{|t|>\frac{d \delta}{4 \pi}}\left(\frac{\delta}{\left(1+\frac{4 \pi^{2} t^{2}}{d^{2} \delta^{2}}\right)^{\frac{d}{4}}}+\frac{(1-\delta)}{\left(1+\frac{4 \pi^{2} t^{2}}{d^{2}(1-\delta)^{2}}\right)^{\frac{d}{4}}}\right)^{4}\left(1+|t|^{d}\right) d t
\end{gathered}
$$


Once we'll show that

$$
\int_{|t|>\frac{d \delta}{4 \pi}}\left(\frac{\delta}{\left(1+\frac{4 \pi^{2} t^{2}}{d^{2} \delta^{2}}\right)^{\frac{d}{4}}}+\frac{(1-\delta)}{\left(1+\frac{4 \pi^{2} t^{2}}{d^{2}(1-\delta)^{2}}\right)^{\frac{d}{4}}}\right)^{4}\left(1+|t|^{d}\right) d t \leq C_{d}
$$

The proof will be done.

Indeed,

$$
\begin{aligned}
& \int_{|t|>\frac{d \delta}{4 \pi}}\left(\frac{\delta}{\left(1+\frac{4 \pi^{2} t^{2}}{d^{2} \delta^{2}}\right)^{\frac{d}{4}}}+\frac{(1-\delta)}{\left(1+\frac{4 \pi^{2} t^{2}}{d^{2}(1-\delta)^{2}}\right)^{\frac{d}{4}}}\right)^{4}\left(1+|t|^{d}\right) d t \\
& \leq \int_{\mathbb{R}}\left(\frac{\delta}{\left(1+\frac{4 \pi^{2} t^{2}}{d^{2} \delta^{2}}\right)^{\frac{d}{4}}}+\frac{(1-\delta)}{\left(1+\frac{4 \pi^{2} t^{2}}{d^{2}(1-\delta)^{2}}\right)^{\frac{d}{4}}}\right)^{4}\left(1+|t|^{d}\right) d t \\
& =\int_{|t| \leq 1}\left(\frac{\delta}{\left(1+\frac{4 \pi^{2} t^{2}}{d^{2} \delta^{2}}\right)^{\frac{d}{4}}}+\frac{(1-\delta)}{\left(1+\frac{4 \pi^{2} t^{2}}{d^{2}(1-\delta)^{2}}\right)^{\frac{d}{4}}}\right)^{4}\left(1+|t|^{d}\right) d t \\
& +\int_{|t|>1}\left(\frac{\delta}{\left(1+\frac{4 \pi^{2} t^{2}}{d^{2} \delta^{2}}\right)^{\frac{d}{4}}}+\frac{(1-\delta)}{\left(1+\frac{4 \pi^{2} t^{2}}{d^{2}(1-\delta)^{2}}\right)^{\frac{d}{4}}}\right)^{4}\left(1+|t|^{d}\right) d t \\
& \leq \int_{|t| \leq 1} 2 d t+\int_{|t|>1}\left(\frac{d^{\frac{d}{2}} \delta^{\frac{d+2}{2}}}{(2 \pi t)^{\frac{d}{2}}}+\frac{d^{\frac{d}{2}}(1-\delta)^{\frac{d+2}{2}}}{(2 \pi t)^{\frac{d}{2}}}\right)^{4} 2|t|^{d} d t \\
& \leq 4+\int_{|t|>1}\left(\frac{d^{\frac{d}{2}}}{(2 \pi t)^{\frac{d}{2}}}+\frac{d^{\frac{d}{2}}}{(2 \pi t)^{\frac{d}{2}}}\right)^{4} 2|t|^{d} d t=4+\frac{2^{5} d^{2 d}}{(2 \pi)^{2 d}} \int_{|t|>1} \frac{d t}{|t|^{d}}=C_{d}
\end{aligned}
$$

Proof of Theorem 3.9. This follows from Lemma 3.10, Lemma 3.11, Lemma 3.12 and the estimation

$$
\left|\widehat{h}^{N}(p, t)-{\widehat{\gamma_{1}}}^{N}(p, t)\right| \leq\left|\widehat{h}(p, t)-\widehat{\gamma_{1}}(p, t)\right| \sum_{k=0}^{N-1}|\widehat{h}(p, t)|^{k}\left|\widehat{\gamma}_{1}(p, t)\right|^{N-k-1}
$$

3.2. small $t$, large $p:|t| \leq \frac{d \delta}{4 \pi}$ and $|p|>\eta$. The main theorem of this subsection is:

Theorem 3.13.

$$
\iint_{|p|>\eta \times|t| \leq \frac{d \delta}{4 \pi}}\left|\widehat{h}^{N}(p, t)-{\widehat{\gamma_{1}}}^{N}(p, t)\right| d p d t \leq \frac{N \delta C_{d} e^{-\frac{(N-2) \eta^{2}}{4 d}}}{N-2}
$$

where $C_{d}$ is a constant depending only on $d$. 
Again, some Lemmas and computations are needed before we can prove the above.

To begin with, we notice that we can't use (3.9) any more as the domain of the $p$ integration changed. Instead, we use the same pre-integration estimation along with Remark 3.8 to find that

$$
\begin{gathered}
\int_{|p|>\eta}|\widehat{h}(p, t)|^{k}\left|\widehat{\gamma_{1}}(p, t)\right|^{N-k-1} d p \\
\leq C_{d} \sum_{j=0}^{k}\left(\begin{array}{c}
k \\
j
\end{array}\right) \frac{\delta^{j}}{\left(1+\frac{\left.4 \pi^{2} t^{2}\right)^{\frac{d j}{4}}}{d^{2} \delta^{2}}\right.} \frac{(1-\delta)^{k-j}}{\left(1+\frac{4 \pi^{2} t^{2}}{d^{2}(1-\delta)^{2}}\right)^{\frac{d(k-j)}{4}}} \\
\cdot \frac{e^{-2 \pi^{2}(N-k-1) \Sigma^{2} t^{2}} e^{-\frac{\pi^{2}\left(\frac{j d \delta}{d^{2} \delta^{2}+4 \pi^{2} t^{2}}+\frac{(k-j) d(1-\delta)}{d^{2}(1-\delta)^{2}+4 \pi^{2} t^{2}}+\frac{N-k-1}{d}\right) \eta^{2}}{2}}}{\left(\frac{j d \delta}{d^{2} \delta^{2}+4 \pi^{2} t^{2}}+\frac{(k-j) d(1-\delta)}{d^{2}(1-\delta)^{2}+4 \pi^{2} t^{2}}+\frac{2(N-k-1)}{d}\right)}
\end{gathered}
$$

We need to justify the usage of the mentioned remark: Since in our domain $|t| \leq$ $\frac{d \delta}{4 \pi}$ we have that

$$
d^{2} \delta^{2}+4 \pi^{2} t^{2} \leq \frac{5 d^{2} \delta^{2}}{4}
$$

Similarly, since $\delta<1-\delta$ we have that

$$
d^{2}(1-\delta)^{2}+4 \pi^{2} t^{2} \leq \frac{5 d^{2}(1-\delta)^{2}}{4}
$$

leading us to conclude that, with the notation of Lemma 3.7:

$$
\begin{gathered}
\alpha=\pi^{2}\left(\frac{j d \delta}{d^{2} \delta^{2}+4 \pi^{2} t^{2}}+\frac{(k-j) d(1-\delta)}{d^{2}(1-\delta)^{2}+4 \pi^{2} t^{2}}+\frac{N-k-1}{d}\right) \\
\geq \pi^{2}\left(\frac{4 j}{5 d \delta}+\frac{4(k-j)}{5 d(1-\delta)}+\frac{N-k-1}{d}\right)
\end{gathered}
$$

if $j \geq 1$ then $\frac{4 j}{5 d \delta} \geq \frac{4}{5 d \delta}>1$ when $\delta$ is small enough. if $k \leq \frac{N}{2}$ then $\frac{N-k-1}{d}>\frac{N-2}{2 d}>1$ for large enough $N$. if $j=0$ and $k>\frac{N}{2}$ then $\frac{4(k-j)}{5 d(1-\delta)} \geq \frac{2 N}{5 d(1-\delta)}>1$ again. in any case, $\alpha>1$. Also, $\beta=\frac{d \delta}{4 \pi}<1$ for $\delta$ small enough and so we managed to justify (3.19). We are now ready to state and prove our first Lemma.

\section{Lemma 3.14.}

$$
\begin{aligned}
\sum_{k=0}^{\left[\frac{N}{2}\right]} \iint_{|p|>\eta \times|t| \leq \frac{d \delta}{4 \pi}} \mid \widehat{h}(p, t) & -\left.\widehat{\gamma_{1}}(p, t)|| \widehat{h}(p, t)\right|^{k}\left|\widehat{\gamma_{1}}(p, t)\right|^{N-k-1} d p d t \\
& \leq \frac{N \delta C_{d} e^{-\frac{(N-2) \eta^{2}}{4 d}}}{N-2}
\end{aligned}
$$

where $C_{d}$ is a constant depending only on d. 
Proof. Since for $k \leq \frac{N}{2}$

$$
\frac{e^{-2 \pi^{2}(N-k-1) \Sigma^{2} t^{2}} e^{-\frac{\pi^{2}\left(\frac{j d \delta}{d^{2} \delta^{2}+4 \pi^{2} t^{2}}+\frac{(k-j) d(1-\delta)}{d^{2}(1-\delta)^{2}+4 \pi^{2} t^{2}}+\frac{N-k-1}{d}\right) \eta^{2}}{2}}}{\left(\frac{j d \delta}{d^{2} \delta^{2}+4 \pi^{2} t^{2}}+\frac{(k-j) d(1-\delta)}{d^{2}(1-\delta)^{2}+4 \pi^{2} t^{2}}+\frac{2(N-k-1)}{d}\right)} \leq \frac{e^{-\frac{(N-2) \eta^{2}}{4 d}}}{\left(\frac{(N-2)}{d}\right)}
$$

we have that due to inequality (3.19)

$$
\begin{gathered}
\sum_{k=0}^{\left[\frac{N}{2}\right]} \iint_{|p|>\eta \times|t| \leq \frac{d \delta}{4 \pi}}\left|\widehat{h}(p, t)-\widehat{\gamma_{1}}(p, t)\right||\widehat{h}(p, t)|^{k}\left|\widehat{\gamma_{1}}(p, t)\right|^{N-k-1} d p d t \\
\leq \frac{2 d C_{d} e^{-\frac{(N-2) \eta^{2}}{4 d}}}{N-2} \sum_{k=0}^{\left.\frac{N}{2}\right]} \int_{|t| \leq \frac{d \delta}{4 \pi}} \sum_{j=0}^{k}\left(\begin{array}{c}
k \\
j
\end{array}\right) \frac{\delta^{j}}{\left(1+\frac{4 \pi^{2} t^{2}}{d^{2} \delta^{2}}\right)^{\frac{d j}{4}}} \frac{(1-\delta)^{k-j}}{\left(1+\frac{4 \pi^{2} t^{2}}{d^{2}(1-\delta)^{2}}\right)^{\frac{d(k-j)}{4}} d t} \\
=\frac{2 d C_{d} e^{-\frac{(N-2) \eta^{2}}{4 d}}}{N-2} \sum_{k=0}^{\left[\frac{N}{2}\right]} \int_{|t| \leq \frac{d \delta}{4 \pi}}\left(\frac{\delta}{\left(1+\frac{4 \pi^{2} t^{2}}{d^{2} \delta^{2}}\right)^{\frac{d}{4}}}+\frac{1-\delta}{\left(1+\frac{4 \pi^{2} t^{2}}{d^{2}(1-\delta)^{2}}\right)^{\frac{d}{4}}}\right)^{k} d t \\
\leq \frac{d N C_{d} e^{-\frac{(N-2) \eta^{2}}{4 d}}}{N-2} \cdot \frac{d \delta}{4 \pi}
\end{gathered}
$$

which concludes the proof.

Next,we notice that

$$
\begin{aligned}
& e^{-\frac{\pi^{2}\left(\frac{j d \delta}{d^{2} \delta^{2}+4 \pi^{2} t^{2}}+\frac{(k-j) d(1-\delta)}{d^{2}(1-\delta)^{2}+4 \pi^{2} t^{2}}+\frac{N-k-1}{d}\right) \eta^{2}}{2}} \\
& \overline{\left(\frac{j d \delta}{d^{2} \delta^{2}+4 \pi^{2} t^{2}}+\frac{(k-j) d(1-\delta)}{d^{2}(1-\delta)^{2}+4 \pi^{2} t^{2}}+\frac{2(N-k-1)}{d}\right)}
\end{aligned}
$$

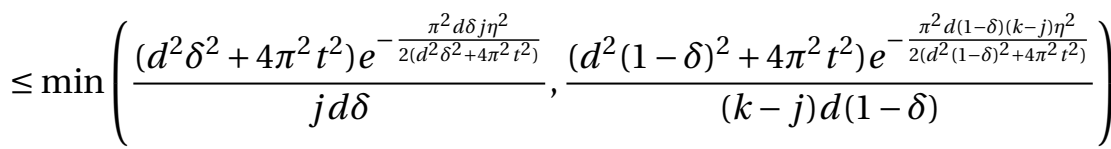

$$
\begin{aligned}
& \leq \min \left(\frac{5 d \delta e^{-\frac{2 \pi^{2} j \eta^{2}}{5 d \delta}}}{4 j}, \frac{5 d(1-\delta) e^{-\frac{2 \pi^{2}(k-j) \eta^{2}}{5 d(1-\delta)}}}{4(k-j)}\right)
\end{aligned}
$$

Thus

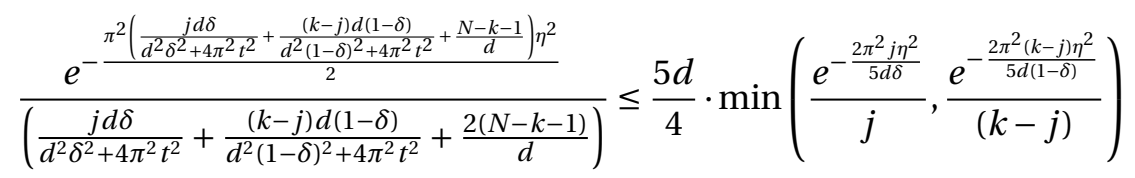

The second Lemma follows:

Lemma 3.15.

$$
\begin{gathered}
\sum_{k=\left[\frac{N}{2}\right]}^{N-1} \iint_{|p|>\eta \times|t| \leq \frac{d \delta}{4 \pi}}\left|\widehat{h}(p, t)-\widehat{\gamma_{1}}(p, t)\right||\widehat{h}(p, t)|^{k}\left|\widehat{\gamma_{1}}(p, t)\right|^{N-k-1} d p d t \\
\leq \frac{N C_{d} \delta e^{-\frac{\pi^{2}(N-2) \eta^{2}}{10 d(1-\delta)}}}{N-2}
\end{gathered}
$$


A COUNTER EXAMPLE TO CERCIGNANI'S CONJECTURE FOR THE D DIMENSIONAL KAC MODEL - AMIT EINAV

where $C_{d}$ is a constant depending only ond.

Proof. Due to inequality (3.19) and (3.21) we find that

$$
\begin{aligned}
& \sum_{k=\left[\frac{N}{2}\right]}^{N-1} \iint_{|p|>\eta \times|t| \leq \frac{d \delta}{4 \pi}}\left|\widehat{h}(p, t)-\widehat{\gamma_{1}}(p, t)\right||\widehat{h}(p, t)|^{k}\left|\widehat{\gamma_{1}}(p, t)\right|^{N-k-1} d p d t \\
& \leq \frac{5 d C_{d}}{2} \sum_{k=\left[\frac{N}{2}\right]}^{N-1} \int_{|t| \leq \frac{d \delta}{4 \pi}} \sum_{j=0}^{k}\left(\begin{array}{c}
k \\
j
\end{array}\right) \frac{\delta^{j}}{\left(1+\frac{4 \pi^{2} t^{2}}{d^{2} \delta^{2}}\right)^{\frac{d j}{4}}} \frac{(1-\delta)^{k-j}}{\left(1+\frac{4 \pi^{2} t^{2}}{d^{2}(1-\delta)^{2}}\right)^{\frac{d(k-j)}{4}}} \cdot \min \left(\frac{e^{-\frac{2 \pi^{2} j \eta^{2}}{5 d \delta}}}{j}, \frac{e^{-\frac{2 \pi^{2}(k-j) \eta^{2}}{5 d(1-\delta)}}}{(k-j)}\right) d t \\
& \leq C_{d} \sum_{k=\left[\frac{N}{2}\right]}^{N-1} \int_{|t| \leq \frac{d \delta}{4 \pi}} \sum_{j=0}^{\left[\frac{k}{2}\right]}\left(\begin{array}{c}
k \\
j
\end{array}\right) \frac{\delta^{j}}{\left(1+\frac{4 \pi^{2} t^{2}}{d^{2} \delta^{2}}\right)^{\frac{d j}{4}}} \frac{(1-\delta)^{k-j}}{\left(1+\frac{4 \pi^{2} t^{2}}{d^{2}(1-\delta)^{2}}\right)^{\frac{d(k-j)}{4}}} \cdot \frac{e^{-\frac{2 \pi^{2}(k-j) \eta^{2}}{5 d(1-\delta)}}}{(k-j)} d t \\
& +C_{d} \sum_{k=\left[\frac{N}{2}\right]}^{N-1} \int_{|t| \leq \frac{d \delta}{4 \pi}} \sum_{j=\left[\frac{k}{2}\right]+1}^{k}\left(\begin{array}{c}
k \\
j
\end{array}\right) \frac{\delta^{j}}{\left(1+\frac{4 \pi^{2} t^{2}}{d^{2} \delta^{2}}\right)^{\frac{d j}{4}}} \frac{(1-\delta)^{k-j}}{\left(1+\frac{4 \pi^{2} t^{2}}{d^{2}(1-\delta)^{2}}\right)^{\frac{d(k-j)}{4}}} \cdot \frac{e^{-\frac{2 \pi^{2} j \eta^{2}}{5 d \delta}}}{j} d t \\
& \leq C_{d} \sum_{k=\left[\frac{N}{2}\right]}^{N-1} \int_{|t| \leq \frac{d \delta}{4 \pi}} \frac{2 e^{-\frac{\pi^{2} k \eta^{2}}{5 d(1-\delta)}}}{k} \sum_{j=0}^{\left[\frac{k}{2}\right]}\left(\begin{array}{c}
k \\
j
\end{array}\right) \frac{\delta^{j}}{\left(1+\frac{4 \pi^{2} t^{2}}{d^{2} \delta^{2}}\right)^{\frac{d j}{4}}} \frac{(1-\delta)^{k-j}}{\left(1+\frac{4 \pi^{2} t^{2}}{d^{2}(1-\delta)^{2}}\right)^{\frac{d(k-j)}{4}}} d t \\
& +C_{d} \sum_{k=\left[\frac{N}{2}\right]}^{N-1} \int_{|t| \leq \frac{d \delta}{4 \pi}} \frac{2 e^{-\frac{\pi^{2} k \eta^{2}}{5 d \delta}}}{k} \sum_{j=\left[\frac{k}{2}\right]+1}^{k}\left(\begin{array}{c}
k \\
j
\end{array}\right) \frac{\delta^{j}}{\left(1+\frac{4 \pi^{2} t^{2}}{d^{2} \delta^{2}}\right)^{\frac{d j}{4}}} \frac{(1-\delta)^{k-j}}{\left(1+\frac{4 \pi^{2} t^{2}}{d^{2}(1-\delta)^{2}}\right)^{\frac{d(k-j)}{4}}} d t \\
& \leq \frac{2 C_{d} e^{-\frac{\pi^{2}(N-2) \eta^{2}}{10 d(1-\delta)}}}{N-2} \sum_{k=\left[\frac{N}{2}\right]}^{N-1} \int_{|t| \leq \frac{d \delta}{4 \pi}} \sum_{j=0}^{k}\left(\begin{array}{c}
k \\
j
\end{array}\right) \frac{\delta^{j}}{\left(1+\frac{4 \pi^{2} t^{2}}{d^{2} \delta^{2}}\right)^{\frac{d j}{4}}} \frac{(1-\delta)^{k-j}}{\left(1+\frac{4 \pi^{2} t^{2}}{d^{2}(1-\delta)^{2}}\right)^{\frac{d(k-j)}{4}}} d t \\
& =\frac{2 C_{d} e^{-\frac{\pi^{2}(N-2) \eta^{2}}{10 d(1-\delta)}}}{N-2} \sum_{k=\left[\frac{N}{2}\right]}^{N-1} \int_{|t| \leq \frac{d \delta}{4 \pi}}\left(\frac{\delta}{\left(1+\frac{4 \pi^{2} t^{2}}{d^{2} \delta^{2}}\right)^{\frac{d}{4}}}+\frac{1-\delta}{\left(1+\frac{4 \pi^{2} t^{2}}{d^{2}(1-\delta)^{2}}\right)^{\frac{d}{4}}}\right)^{k} d t
\end{aligned}
$$

from which the result follows.

Proof of Theorem 3.13. This follows from Lemma 3.14, Lemma 3.15, the fact that $\frac{\pi^{2}}{10(1-\delta)}>\frac{1}{4}$ and the inequality mentioned at the proof of Theorem 3.9.

3.3. smallish $t$, small $p$ : $\frac{d \delta^{1+\beta}}{4 \pi}<|t| \leq \frac{d \delta}{4 \pi}$ and $|p| \leq \eta$. The main theorem of this subsection is the following:

Theorem 3.16.

$$
\begin{gathered}
\iint_{|p| \leq \eta \times \frac{d \delta^{1+\beta}}{4 \pi}<|t| \leq \frac{d \delta}{4 \pi}}\left|\widehat{h}^{N}(p, t)-\widehat{\gamma}^{N}(p, t)\right| d p d t \\
\left.\leq \frac{2^{d} \eta^{d} N d \delta}{4 \pi} e^{-\frac{d^{2}(N-2) \Sigma^{2} \delta^{2+2 \beta}}{16}}+2^{d} \eta^{d} N\left(1-\frac{d \delta^{1+2 \beta}}{16}+\delta^{1+4 \beta} \xi(\delta)\right)\right)^{\frac{N}{2}}
\end{gathered}
$$


where $\xi$ is analytic in $|x|<\frac{1}{2}$.

We start by noticing that

$$
\begin{aligned}
& \iint_{|p|<\eta \times \frac{d \delta^{1+\beta}}{4 \pi}<|t| \leq \frac{d \delta}{4 \pi}}\left|\widehat{h}(p, t)-\widehat{\gamma_{1}}(p, t)\right| \sum_{k=0}^{N-1}|\widehat{h}(p, t)|^{k}\left|\widehat{\gamma_{1}}(p, t)\right|^{N-1-k} d p d t \\
& \leq 2(2 \eta)^{d} \int_{\frac{d \delta^{1+\beta}}{4 \pi}<|t| \leq \frac{d \delta}{4 \pi}}\left(\frac{\delta}{\left(1+\frac{4 \pi^{2} t^{2}}{d^{2} \delta^{2}}\right)^{\frac{d}{4}}}+\frac{1-\delta}{\left(1+\frac{4 \pi^{2} t^{2}}{d^{2}(1-\delta)^{2}}\right)^{\frac{d}{4}}}\right)^{k} e^{-2 \pi^{2}(N-k-1) \Sigma^{2} t^{2} d t}
\end{aligned}
$$

Another observation we make is the following: In our domain

$$
\begin{gathered}
\frac{\delta}{\left(1+\frac{4 \pi^{2} t^{2}}{d^{2} \delta^{2}}\right)^{\frac{d}{4}}}+\frac{1-\delta}{\left(1+\frac{4 \pi^{2} t^{2}}{d^{2}(1-\delta)^{2}}\right)^{\frac{d}{4}}} \leq \frac{\delta}{\left(1+\frac{\delta^{2 \beta}}{4}\right)^{\frac{d}{4}}}+\frac{1-\delta}{\left(1+\frac{\delta^{2+2 \beta}}{4(1-\delta)^{2}}\right)^{\frac{d}{4}}} \\
=\delta\left(1-\frac{d \delta^{2 \beta}}{16}+\delta^{4 \beta} \phi\left(\delta^{2 \beta}\right)\right)+(1-\delta)\left(1-\frac{d \delta^{2+2 \beta}}{16(1-\delta)^{2}}+\frac{\delta^{4+4 \beta}}{(1-\delta)^{4}} \phi\left(\frac{\delta^{2+2 \beta}}{(1-\delta)^{2}}\right)\right)
\end{gathered}
$$

where $\phi$ in analytic in $|x|<\frac{1}{2}$. Opening the parenthesis leads to

$$
=1-\frac{d \delta^{1+2 \beta}}{16}-\frac{d \delta^{2+2 \beta}}{16(1-\delta)}+\delta^{1+4 \beta} \phi\left(\delta^{2 \beta}\right)+\frac{\delta^{4+4 \beta}}{(1-\delta)^{3}} \phi\left(\frac{\delta^{2+2 \beta}}{(1-\delta)^{2}}\right)
$$

which we can write as the inequality:

$$
\frac{\delta}{\left(1+\frac{4 \pi^{2} t^{2}}{d^{2} \delta^{2}}\right)^{\frac{d}{4}}}+\frac{1-\delta}{\left(1+\frac{4 \pi^{2} t^{2}}{d^{2}(1-\delta)^{2}}\right)^{\frac{d}{4}}} \leq 1-\frac{d \delta^{1+2 \beta}}{16}+\delta^{1+4 \beta} \xi(\delta)
$$

where $\xi$ is analytic in $|x|<\frac{1}{2}$.

We are now ready to state and prove this subsection's Lemmas.

\section{Lemma 3.17.}

$$
\begin{aligned}
\sum_{k=0}^{\left[\frac{N}{2}\right]} \iint_{|p|<\eta \times \frac{d \delta^{1+\beta}}{4 \pi}<|t| \leq \frac{d \delta}{4 \pi}}\left|\widehat{h}(p, t)-\widehat{\gamma_{1}}(p, t)\right||\widehat{h}(p, t)|^{k}\left|\widehat{\gamma_{1}}(p, t)\right|^{N-1-k} d p d t \\
\leq \frac{2^{d} \eta^{d} N d \delta}{4 \pi} e^{-\frac{d^{2}(N-2) \Sigma^{2} \delta^{2+2 \beta}}{16}}
\end{aligned}
$$

Proof. Using inequality (3.24) along with the fact that $|\widehat{h}(p, t)| \leq 1$ leads us to conclude that

$$
\begin{gathered}
\sum_{k=0}^{\left[\frac{N}{2}\right]} \iint_{|p|<\eta \times \frac{d \delta^{1+\beta}}{4 \pi}<|t| \leq \frac{d \delta}{4 \pi}}\left|\widehat{h}(p, t)-\widehat{\gamma_{1}}(p, t)\right||\widehat{h}(p, t)|^{k}\left|\widehat{\gamma_{1}}(p, t)\right|^{N-1-k} d p d t \\
\leq 2^{d+1} \eta^{d} \sum_{k=0}^{\left[\frac{N}{2}\right]} \int_{\frac{d \delta^{1+\beta}}{4 \pi}<|t| \leq \frac{d \delta}{4 \pi}} e^{-2 \pi^{2}(N-k-1) \Sigma^{2} t^{2}} d t
\end{gathered}
$$


A COUNTER EXAMPLE TO CERCIGNANI'S CONJECTURE FOR THE D DIMENSIONAL KAC MODEL - AMIT EINAY

$$
\begin{gathered}
\leq 2^{d+1} \eta^{d} \sum_{k=0}^{\left[\frac{N}{2}\right]} \int_{\frac{d \delta^{1+\beta}}{4 \pi}<|t| \leq \frac{d \delta}{4 \pi}} e^{-\pi^{2}(N-2) \Sigma^{2} t^{2}} d t \leq 2^{d} \eta^{d} N \int_{\frac{d \delta^{1+\beta}}{4 \pi}<|t| \leq \frac{d \delta}{4 \pi}} e^{-\pi^{2}(N-2) \Sigma^{2} t^{2}} d t \\
\leq \frac{2^{d} \eta^{d} N d \delta}{4 \pi} e^{-\frac{d^{2}(N-2) \Sigma^{2} \delta^{2+2 \beta}}{16}}
\end{gathered}
$$

and the proof is complete.

\section{Lemma 3.18.}

$$
\begin{aligned}
& \sum_{k=\left[\frac{N}{2}\right]+1}^{N-1} \iint_{|p|<\eta \times \frac{d \delta^{1}+\beta}{4 \pi}<|t| \leq \frac{d \delta}{4 \pi}}\left|\widehat{h}(p, t)-\widehat{\gamma_{1}}(p, t)\right||\widehat{h}(p, t)|^{k}\left|\widehat{\gamma_{1}}(p, t)\right|^{N-1-k} d p d t \\
& \leq 2^{d} \eta^{d} N\left(1-\frac{d \delta^{1+2 \beta}}{16}+\delta^{1+4 \beta} \xi(\delta)\right)^{\frac{N}{2}}
\end{aligned}
$$

where $\xi$ in analytic in $|x|<\frac{1}{2}$.

Proof. Using inequality (3.24) and (3.25) we find that

$$
\begin{gathered}
\sum_{k=\left[\frac{N}{2}\right]+1}^{N-1} \iint_{|p|<\eta \times \frac{d \delta^{1+\beta}}{4 \pi}<|t| \leq \frac{d \delta}{4 \pi}}\left|\widehat{h}(p, t)-\widehat{\gamma_{1}}(p, t)\right||\widehat{h}(p, t)|^{k}\left|\widehat{\gamma_{1}}(p, t)\right|^{N-1-k} d p d t \\
\leq 2^{d+1} \eta^{d} \sum_{k=\left[\frac{N}{2}\right]+1}^{N-1} \int_{\frac{d \delta^{1+\beta}}{4 \pi}<|t| \leq \frac{d \delta}{4 \pi}}\left(\frac{\delta}{\left(1+\frac{4 \pi^{2} t^{2}}{d^{2} \delta^{2}}\right)^{\frac{d}{4}}}+\frac{1-\delta}{\left(1+\frac{4 \pi^{2} t^{2}}{d^{2}(1-\delta)^{2}}\right)^{\frac{d}{4}}}\right)^{k} d t \\
\leq 2^{d} \eta^{d} N\left(1-\frac{d \delta^{1+2 \beta}}{16}+\delta^{1+4 \beta} \xi(\delta)\right)^{\frac{N}{2}}
\end{gathered}
$$

which completes the proof.

Proof of Theorem 3.16. Much like previous theorems, this is a direct result of Lemma 3.17 and Lemma 3.18.

3.4. Tiny $t$, small $p:|t|<\frac{d \delta^{1+\beta}}{4 \pi}$ and $|p| \leq \eta=\delta^{\frac{1}{2}+\beta}$. The main result of this subsection is

\section{Theorem 3.19.}

$$
\begin{gathered}
\iint_{|p| \leq \delta^{\frac{1}{2}+\beta} \times|t| \leq \frac{d \delta^{1+\beta}}{4 \pi}}\left|\widehat{h}^{N}(p, t)-{\widehat{\gamma_{1}}}^{N}(p, t)\right| d p d t \\
\quad \leq \frac{C_{d}}{\Sigma} \delta^{\frac{3}{2}+4 \beta+\frac{d}{2}+d \beta}+\frac{C_{d} \sqrt{N}}{\Sigma} \delta^{1+3 \beta+\frac{d}{2}+d \beta}
\end{gathered}
$$

where $C_{d}$ is a constant depending only on $d$.

We start by the simple observation that in this domain

$$
\left|\Sigma^{2} t\right| \leq \frac{(d+2) \delta^{\beta}}{16 \pi(1-\delta)}<\frac{(d+2) \delta^{\beta}}{8 \pi}
$$


when $\delta<\frac{1}{2}$.

The main difficulty in our domain is the need to have a more precise approximation to the functions involved. We start with the easier amongst the two:

\section{Lemma 3.20.}

$$
\widehat{\gamma_{1}}(p, t)=\left(1-2 \pi i t-2 \pi^{2} t^{2}\left(\Sigma^{2}+1\right)+t^{3} g(t)\right)\left(1-\frac{2 \pi^{2}|p|^{2}}{d}+|p|^{4} f\left(|p|^{2}\right)\right)
$$

where $g, f$ are entire and there exist constants $M_{0}, M_{1}$, depending only on $d$, such that

$$
\begin{gathered}
|g(t)| \leq M_{0}+\frac{M_{1}}{\delta} \\
\left|f\left(|p|^{2}\right)\right| \leq M_{0}
\end{gathered}
$$

Proof. Using the approximation $e^{x}=1+x+\frac{x^{2}}{2}+x^{3} \phi(x)$, where $\phi$ is entire, we find that

$$
\begin{aligned}
e^{-\frac{2 \pi^{2}|p|^{2}}{d}} & =1-\frac{2 \pi^{2}|p|^{2}}{d}+\frac{4 \pi^{4}|p|^{4}}{d^{2}} \phi_{1}\left(\frac{2 \pi^{2}|p|^{2}}{d}\right) \\
e^{-2 \pi i t} & =1-2 \pi i t-\frac{4 \pi^{2} t^{2}}{2}-8 \pi^{3} t^{3} \phi(2 \pi i t)
\end{aligned}
$$

and

$$
e^{-2 \pi^{2} \Sigma^{2} t^{2}}=1-2 \pi^{2} \Sigma^{2} t^{2}+\frac{4 \pi^{4} \Sigma^{4} t^{4}}{2}+8 \pi^{6} \Sigma^{6} t^{6} \phi\left(2 \pi^{2} \Sigma^{2} t^{2}\right)
$$

where $\phi_{1}$ is entire. Thus

$$
\begin{gathered}
e^{-2 \pi i t} \cdot e^{-2 \pi^{2} \Sigma^{2} t^{2}}=1-2 \pi i t-2 \pi^{2} t^{2}\left(\Sigma^{2}+1\right)+\pi^{3} t^{3}\left(4 i \Sigma^{2}-8 \phi(2 \pi i t)\right) \\
+4 \pi^{4} \Sigma^{2} t^{4}+16 \pi^{5} t^{5} \Sigma^{2} \phi(2 \pi i t)+\pi^{4} t^{4}\left(2 \Sigma^{4}+8 \pi^{2} \Sigma^{6} t^{2} \phi\left(2 \pi^{2} \Sigma^{2} t^{2}\right)\right) e^{-2 \pi i t} \\
=1-2 \pi i t-2 \pi^{2} t^{2}\left(\Sigma^{2}+1\right)+t^{3} g(t)
\end{gathered}
$$

We clearly have that $g(t)$ is entire, and

$$
\begin{gathered}
|g(t)| \leq 4 \pi^{3} \Sigma^{2}+8 \pi^{3}|\phi(2 \pi i t)|+4 \pi^{4} \Sigma^{2}|t|+16 \pi^{5} t^{2} \Sigma^{2}|\phi(2 \pi i t)|+2 \pi^{4}|t| \Sigma^{4} \\
+8 \pi^{6}|t|^{3} \Sigma^{6}\left|\phi\left(2 \pi^{2} \Sigma^{2} t^{2}\right)\right| \leq \frac{2 \pi^{3}(d+2)}{d \delta}+8 \pi^{3} M_{\text {sup }}+\frac{\pi^{3}(d+2) \delta^{\beta}}{2}+\frac{\pi^{3} d(d+2) \delta^{1+2 \beta} M_{\text {sup }}}{2} \\
+\frac{\pi^{4}(d+2)^{2} \delta^{\beta}}{8 d \delta}+\frac{\pi^{3}(d+2)^{3} \delta^{3 \beta} M_{\text {sup }}}{64}
\end{gathered}
$$

where $M_{\text {sup }}=\sup _{|x|<1}|\phi(x)|$. A simpler argument on $f$ leads to the desired result.

Next, we find an approximation to $\widehat{h}(p, t)$

\section{Lemma 3.21.}

$\widehat{h}(p, t)=1-2 \pi i t-2 \pi^{2} t^{2}\left(\Sigma^{2}+1\right)+t^{3} h(t)-\frac{2 \pi^{2}|p|^{2}}{d}-\frac{\pi^{2}|p|^{2}}{d} t h_{1}(t)+|p|^{4} h_{2}(p, t)$ 
where $h, h_{1}, h_{2}$ are analytic in the domain and there exist constants $M_{0}, M_{1}, M_{2}$, independent in $\delta$, such that

$$
\begin{gathered}
|h(t)| \leq M_{0}+\frac{M_{2}}{\delta^{2}} \\
\left|h_{1}(t)\right| \leq M_{0}+\frac{M_{1}}{\delta} \\
\left|h_{2}(p, t)\right| \leq\left(M_{0}+\frac{M_{1}}{\delta}\right) M_{p . \delta}
\end{gathered}
$$

with $M_{p, \delta}=\sup _{|x| \leq \frac{\pi^{2}|p|^{2}}{d \delta}}|\phi(x)|$ and $\phi$ entire.

Proof. Using the exponential approximation we find that

$$
\frac{e^{-\frac{\pi^{2}|p|^{2}}{d+\frac{2 \pi i t}{d \delta}}}}{\left(1+\frac{2 \pi i t}{d \delta}\right)^{\frac{d}{2}}}=\frac{1}{\left(1+\frac{2 \pi i t}{d \delta}\right)^{\frac{d}{2}}}-\frac{\pi^{2}|p|^{2}}{d \delta\left(1+\frac{2 \pi i t}{d \delta}\right)^{\frac{d+2}{2}}}+\frac{\pi^{4}|p|^{4}}{d^{2} \delta^{2}\left(1+\frac{2 \pi i t}{d \delta}\right)^{\frac{d+4}{2}}} \phi\left(\frac{\pi^{2}|p|^{2}}{d \delta+2 \pi i t}\right)
$$

Another approximation we will need to use is the following:

$$
\frac{1}{(1+x)^{\alpha}}=1-\alpha x+\frac{\alpha(\alpha+1)}{2} x^{2}+x^{3} \cdot g_{\alpha}(x)
$$

where $g_{\alpha}(x)$ is analytic in $|x|<1$.

We conclude that

and so

$$
\begin{gathered}
\frac{1}{\left(1+\frac{2 \pi i t}{d \delta}\right)^{\frac{d}{2}}}=1-\frac{\pi i t}{\delta}-\frac{(d+2)}{4 d \delta^{2}} \cdot 2 \pi^{2} t^{2}-\frac{8 \pi^{3} i t^{3}}{d^{3} \delta^{3}} g_{\frac{d}{2}}\left(\frac{2 \pi i t}{d \delta}\right) \\
\frac{1}{\left(1+\frac{2 \pi i t}{d \delta}\right)^{\frac{d+2}{2}}}=1+\frac{(d+2) \pi i t}{d \delta} g_{1}\left(\frac{2 \pi i t}{d \delta}\right) \\
\frac{1}{\left(1+\frac{2 \pi i t}{d \delta}\right)^{\frac{d+4}{2}}}=1+\frac{(d+4) \pi i t}{d \delta} g_{2}\left(\frac{2 \pi i t}{d \delta}\right)
\end{gathered}
$$

$$
\begin{gathered}
\frac{\delta}{\left(1+\frac{2 \pi i t}{d \delta}\right)^{\frac{d}{2}}}+\frac{(1-\delta)}{\left(1+\frac{2 \pi i t}{d(1-\delta)}\right)^{\frac{d}{2}}}=1-2 \pi i t-2 \pi^{2} t^{2}\left(\Sigma^{2}+1\right)+\frac{8 \pi^{3} i t^{3}}{d^{3}}\left(\frac{g_{\frac{d}{2}}\left(\frac{2 \pi i t}{d \delta}\right)}{\delta^{2}}+\frac{g_{\frac{d}{2}}\left(\frac{2 \pi i t}{d(1-\delta)}\right)}{(1-\delta)^{2}}\right) \\
=1-2 \pi i t-2 \pi^{2} t^{2}\left(\Sigma^{2}+1\right)+t^{3} h(t)
\end{gathered}
$$

where

$$
|h(t)| \leq \frac{8 \pi^{3}}{d^{3}}\left(\frac{M_{\text {sup }}}{\delta^{2}}+\frac{M_{\text {sup }}}{(1-\delta)^{2}}\right)
$$

and $M_{\text {sup }}=\sup _{|x|<\frac{1}{2}}\left|g_{\frac{d}{2}}(x)\right|$.

Next, we see that

$$
-\frac{\pi^{2}|p|^{2}}{d\left(1+\frac{2 \pi i t}{d \delta}\right)^{\frac{d+2}{2}}}=-\frac{\pi^{2}|p|^{2}}{d}\left(1+\frac{(d+2) \pi i t}{d \delta} g_{1}\left(\frac{2 \pi i t}{d \delta}\right)\right)
$$


leading to

$$
-\frac{\pi^{2}|p|^{2}}{d\left(1+\frac{2 \pi i t}{d \delta}\right)^{\frac{d+2}{2}}}-\frac{\pi^{2}|p|^{2}}{d\left(1+\frac{2 \pi i t}{d(1-\delta)}\right)^{\frac{d+2}{2}}}=-\frac{2 \pi^{2}|p|^{2}}{d}-\frac{\pi^{2}|p|^{2}}{d} \cdot t h_{1}(t)
$$

with

$$
\left|h_{1}(t)\right| \leq \frac{(d+2) \pi M_{1, s u p}}{d \delta(1-\delta)}
$$

and $M_{1, \text { sup }}=\sup _{|x|<\frac{1}{2}}\left|g_{1}(x)\right|$.

Lastly,

$$
\begin{aligned}
\frac{\pi^{4}|p|^{4}}{d^{2} \delta\left(1+\frac{2 \pi i t}{d \delta}\right)^{\frac{d+4}{2}}} \phi\left(\frac{\pi^{2}|p|^{2}}{d \delta+2 \pi i t}\right) & +\frac{\pi^{4}|p|^{4}}{d^{2}(1-\delta)\left(1+\frac{2 \pi i t}{d(1-\delta)}\right)^{\frac{d+4}{2}}} \phi\left(\frac{\pi^{2}|p|^{2}}{d(1-\delta)+2 \pi i t}\right) \\
& =|p|^{4} h_{2}(p, t)
\end{aligned}
$$

where

$$
\begin{gathered}
\left|h_{2}(p, t)\right| \leq \frac{\pi^{4}}{d^{2} \delta} \phi\left(\frac{\pi^{2}|p|^{2}}{d \delta+2 \pi i t}\right)+\frac{\pi^{4}}{d^{2}(1-\delta)} \phi\left(\frac{\pi^{2}|p|^{2}}{d(1-\delta)+2 \pi i t}\right) \\
\leq \frac{\pi^{4} M_{p, \delta}}{d^{2} \delta(1-\delta)}
\end{gathered}
$$

and $M_{p, \delta}=\sup _{|x| \leq \frac{\pi^{2}|p|^{2}}{d \delta}}|\phi(x)|$. The result follows readily from all the above estimations.

Combining the two last Lemmas yields the following:

Lemma 3.22. When $|t|<\frac{d \delta^{1+\beta}}{4 \pi}$ and $|p| \leq \delta^{\frac{1}{2}+\beta}$ we have that there exist constants $M_{0}, M_{1}, M_{2}$, independent of $\delta$, such that

$\left|\widehat{h}(p, t)-\widehat{\gamma_{1}}(p, t)\right| \leq|t|^{3}\left(M_{0}+\frac{M_{1}}{\delta}+\frac{M_{2}}{\delta^{2}}\right)+\frac{\pi^{2}|p|^{2}|t|}{d}\left(M_{0}+\frac{M_{1}}{\delta}\right)+|p|^{4}\left(M_{0}+\frac{M_{1}}{\delta}\right)$

Proof. We can rewrite equation (3.29) as

$1-2 \pi i t-2 \pi^{2} t^{2}\left(\Sigma^{2}+1\right)+t^{3} g(t)-\frac{2 \pi^{2}|p|^{2}}{d}-\frac{\pi^{2}|p|^{2}}{d} t q_{1}(t)+|p|^{4} f\left(|p|^{2}\right) e^{-2 \pi^{2} \Sigma^{2} t^{2}} e^{-2 \pi i t}$

with $q_{1}(t)=-4 \pi i-4 \pi^{2} t\left(\Sigma^{2}+1\right)+2 t^{2} g(t)$. By the conditions on the domain and $g$ we know that $\left|q_{1}(t)\right| \leq M_{0}$ for some constant.

Combining this with equation (3.30) and using the same notations as in the approximation Lemmas, we find that

$$
\begin{gathered}
\left|\widehat{h}(p, t)-\widehat{\gamma_{1}}(p, t)\right| \leq|t|^{3}(|g(t)|+|h(t)|)+\frac{\pi^{2}|p|^{2}|t|}{d}\left(\left|q_{1}(t)\right|+\left|h_{1}(t)\right|\right) \\
+|p|^{4}\left(|f(p)|+\left|h_{2}(p, t)\right|\right)
\end{gathered}
$$


Since $\eta=\delta^{\frac{1}{2}+\beta}$ we have that $M_{p, \delta} \leq \sup _{|x| \leq \frac{\pi^{2} \delta^{2} \beta}{d}}|\phi(x)| \leq M_{0}$, and so we can find constants $M_{0}, M_{1}, M_{2}$ such that

$\left|\widehat{h}(p, t)-\widehat{\gamma_{1}}(p, t)\right| \leq|t|^{3}\left(M_{0}+\frac{M_{1}}{\delta}+\frac{M_{2}}{\delta^{2}}\right)+\frac{\pi^{2}|p|^{2}|t|}{d}\left(M_{0}+\frac{M_{1}}{\delta}\right)+|p|^{4}\left(M_{0}+\frac{M_{1}}{\delta}\right)$

which is the desired result.

Proof of Theorem 3.19. Since

$$
\begin{gathered}
\iint_{|p| \leq \delta^{\frac{1}{2}+\beta} \times|t| \leq \frac{d \delta^{1+\beta}}{4 \pi}}\left|\widehat{h}(p, t)-\widehat{\gamma_{1}}(p, t)\right||\widehat{h}(p, t)|^{N-1} d p d t \\
\quad \leq \iint_{|p| \leq \delta^{\frac{1}{2}+\beta} \times|t| \leq \frac{d \delta^{1+\beta}}{4 \pi}}\left|\widehat{h}(p, t)-\widehat{\gamma_{1}}(p, t)\right| d p d t
\end{gathered}
$$

inequality (3.31) shows that the above expression is bounded by

$$
\begin{aligned}
\left(M_{0}+\frac{M_{1}}{\delta}+\frac{M_{2}}{\delta^{2}}\right) \cdot \delta^{4+4 \beta} \cdot \delta^{\frac{d}{2}+d \beta} & +\left(M_{0}+\frac{M_{1}}{\delta}\right) \cdot \delta^{2+2 \beta} \cdot \delta^{\frac{d}{2}+d \beta+1+2 \beta}+\left(M_{0}+\frac{M_{1}}{\delta}\right) \cdot \delta^{1+\beta} \cdot \delta^{\frac{d}{2}+d \beta+2+4 \beta} \\
& \leq \frac{C_{d}}{\Sigma} \delta^{\frac{3}{2}+4 \beta+\frac{d}{2}+d \beta}
\end{aligned}
$$

By Lemma 3.6 we find that

$$
\begin{gathered}
\sum_{k=0}^{N-2} \int_{|t| \leq \frac{d \delta^{1+\beta}}{4 \pi}} e^{-2 \pi^{2}(N-k-1) \Sigma^{2} t^{2}} d t=\sum_{k=1}^{N-1} \int_{|t| \leq \frac{d \delta^{1+\beta}}{4 \pi}} e^{-2 \pi^{2} k \Sigma^{2} t^{2}} d t \leq \sqrt{\pi} \sum_{k=1}^{N-1} \frac{\sqrt{1-e^{-\frac{d^{2} \Sigma^{2} k \delta^{2+2 \beta}}{4}}}}{\sqrt{2 \pi^{2} \Sigma^{2} k}} \\
\leq \frac{C_{d}}{\sum} \sum_{k=1}^{N-1} \frac{1}{\sqrt{k}} \leq \frac{C_{d} \sqrt{N}}{\Sigma}
\end{gathered}
$$

and since on our domain

$$
\left|\widehat{h}(p, t)-\widehat{\gamma_{1}}(p, t)\right| \leq C_{d} \delta^{1+3 \beta}
$$

we find that

$$
\begin{gathered}
\sum_{k=0}^{N-2} \iint_{|p| \leq \delta^{\frac{1}{2}+\beta} \times|t| \leq \frac{d \delta^{1+\beta}}{4 \pi}}\left|\widehat{h}(p, t)-\widehat{\gamma_{1}}(p, t)\right||\widehat{h}(p, t)|^{k}\left|\widehat{\gamma_{1}}(p, t)\right|^{N-k-1} d p d t \\
C_{d} \delta^{1+3 \beta} \sum_{k=0}^{N-2} \iint_{|p| \leq \delta^{\frac{1}{2}+\beta} \times|t| \leq \frac{d \delta^{1+\beta}}{4 \pi}} e^{-2 \pi^{2}(N-k-1) \Sigma^{2} t^{2}} d p d t \leq \frac{C_{d} \sqrt{N}}{\Sigma} \delta^{1+3 \beta+\frac{d}{2}+d \beta}
\end{gathered}
$$

which finishes the proof.

Now that we have all the domains sorted we can combine all the theorems into an appropriate approximation theorem. 


\subsection{The proof of the main approximation theorem.}

Theorem 3.23. For any $\beta>0$ and $0<\delta<\frac{1}{2}$ small enough we have that

$$
\begin{gathered}
\iint_{\mathbb{R}^{d} \times \mathbb{R}}\left|\widehat{h}^{N}(p, t)-\widehat{\gamma}^{N}(p, t)\right| d p d t \leq \frac{C_{d}}{N^{\frac{d}{2}} \Sigma} \cdot e^{-\frac{(d+2-4(1-\delta) \delta d)(N-2) \delta}{128(1-\delta)}} \\
+\frac{N C_{d}}{2 \Sigma}\left(1-\left(1-\left(\frac{4}{5}\right)^{\frac{d}{4}}\right) \delta+\delta^{2} \varphi(\delta)\right)^{\frac{N}{2}} \cdot e^{-\frac{d(d+2-4(\delta(1-\delta) \delta}{128(1-\delta)}}+C_{d}\left(1-\left(1-\left(\frac{4}{5}\right)^{\frac{d}{4}}\right) \delta+\delta^{2} \varphi(\delta)\right)^{N-5} \\
\delta C_{d} e^{-\frac{(N-2) 1^{1+2 \beta}}{4 d}}+C_{d} N \delta^{1+\frac{d}{2}+d \beta} e^{-\frac{d(N-2) \delta^{1+2 \beta}(d+2-4 d \delta(1-\delta))}{64(1-\delta)}} \\
+2^{d} \delta^{\frac{d}{2}+d \beta} N\left(1-\frac{d \delta^{1+2 \beta}}{16}+\delta^{1+4 \beta} \xi(\delta)\right)^{\frac{N}{2}}+\frac{C_{d}}{\Sigma} \delta^{\frac{3}{2}+4 \beta+\frac{d}{2}+d \beta}+\frac{C_{d} \sqrt{N}}{\Sigma} \delta^{1+3 \beta+\frac{d}{2}+d \beta} \\
=\frac{\epsilon(N)}{\Sigma N^{\frac{d+1}{2}}}
\end{gathered}
$$

where $C_{d}$ is a constant depending only on $d$, and $\varphi$ and $\xi$ are analytic in $|x|<\frac{1}{2}$.

Proof. This follows immediately from Theorems 3.9, 3.13, 3.16 and 3.19.

Proof of Theorem 3.4. We notice that the theorem is equivalent to showing that

$$
\sup _{v \in \mathbb{R}^{d}, u \in \mathbb{R}}\left|h^{* N}(u, v)-\gamma_{N}(u, v)\right| \leq \frac{\epsilon(N)}{\sum N^{\frac{d+1}{2}}}
$$

with $\lim _{N \rightarrow \infty} \epsilon(N)=0$.

Since

$$
\sup _{\nu \in \mathbb{R}^{d}, u \in \mathbb{R}}\left|h^{* N}(u, v)-\gamma_{N}(u, v)\right| \leq \iint_{\mathbb{R}^{d} \times \mathbb{R}}\left|\widehat{h}^{N}(p, t)-{\widehat{\gamma_{1}}}^{N}(p, t)\right| d p d t
$$

we only need to show that the specific choice of $\delta_{N}$ will give $\epsilon(N)$ that goes to zero, in the notations of Theorem 3.23.

This will be true if we have the following conditions:
i. $\delta_{N}^{1+2 \beta} N \underset{N \rightarrow \infty}{\longrightarrow} \infty$
ii. $N^{\frac{d+1}{2}} \delta_{N}^{\frac{3}{2}+4 \beta+\frac{d}{2}+d \beta} \underset{N \rightarrow \infty}{\longrightarrow} 0$
iii. $N^{\frac{d}{2}+1} \delta_{N}^{1+3 \beta+\frac{d}{2}+d \beta} \underset{N \rightarrow \infty}{\longrightarrow} 0$

The choice $\delta_{N}=\frac{1}{N^{1-\eta} \beta}$ with

$$
\frac{2 \beta}{1+2 \beta}<\eta_{\beta}<\frac{(3+d) \beta}{1+3 \beta+\frac{d}{2}+d \beta}
$$

will satisfy all the conditions (and it is possible to prove that $\frac{2 \beta}{1+2 \beta}<\frac{(3+d) \beta}{1+3 \beta+\frac{d}{2}+d \beta}$ ). Indeed,

$$
\frac{N}{N^{(1-\eta)(1+2 \beta)}}=N^{\eta(1+2 \beta)-2 \beta}
$$


thus, in order to get the first condition we must have $\eta>\frac{2 \beta}{1+2 \beta}$.

Next we notice that

$$
N^{\frac{d+1}{2}} N^{(\eta-1)\left(\frac{3}{2}+4 \beta+\frac{d}{2}+d \beta\right)}=N^{\eta\left(\frac{3}{2}+4 \beta+\frac{d}{2}+d \beta\right)-(1+4 \beta+d \beta)}
$$

so the second condition amounts to

$$
\eta<\frac{1+4 \beta+d \beta}{\frac{3}{2}+4 \beta+\frac{d}{2}+d \beta}
$$

which will obviously be satisfied for small enough $\beta$ and won't contradict the first one.

Lastly,

$$
N^{\frac{d}{2}+1} N^{(\eta-1)\left(1+3 \beta+\frac{d}{2}+d \beta\right)}=N^{\eta\left(1+3 \beta+\frac{d}{2}+d \beta\right)-(3+d) \beta}
$$

so the third condition amounts to

$$
\eta<\frac{(3+d) \beta}{1+3 \beta+\frac{d}{2}+d \beta}
$$

In order to be consistent we must verify that

$$
\frac{2 \beta}{1+2 \beta}<\frac{(3+d) \beta}{1+3 \beta+\frac{d}{2}+d \beta}
$$

This is equivalent to

$$
2+6 \beta+d+2 d \beta<(3+d)(1+2 \beta)=3+d+6 \beta+2 d \beta
$$

which is equivalent to $2<3$ and the proof is complete.

\section{THE MAIN RESUlT}

We're finally ready to prove Theorem 1.1. This will consist of two theorems, one dealing with the denominator of (1.5) and one with its numerator. Throughout this section the function $F_{N}$ will be defined as

$$
F_{N}\left(v_{1}, \ldots, v_{N}\right)=\frac{\prod_{i=1}^{N} f_{\delta_{N}}\left(v_{i}\right)}{Z_{B}^{N}\left(f_{\delta_{N}}, \sqrt{N}, 0\right)}
$$

\section{Theorem 4.1.}

$$
\lim _{N \rightarrow \infty} \frac{H_{N}\left(F_{N}\right)}{N}=\frac{d \log 2}{2}
$$

Proof. By the definition

$$
\begin{gathered}
H_{N}\left(F_{N}\right)=\frac{1}{\mathcal{Z}_{B}^{N}\left(f_{\delta_{N}}, \sqrt{N}, 0\right)} \int_{\mathscr{S}_{B}^{N}(N, 0)} \prod_{i=1}^{N} f_{\delta_{N}}\left(v_{i}\right) \log \left(\prod_{i=1}^{N} f_{\delta_{N}}\left(v_{i}\right)\right) d \sigma_{N, 0}^{N}-\log \left(Z_{B}^{N}\left(f_{\delta_{N}}, \sqrt{N}, 0\right)\right) \\
=\frac{N}{\mathcal{Z}_{B}^{N}\left(f_{\delta_{N}}, \sqrt{N}, 0\right)} \int_{\mathscr{S}_{B}^{N}(N, 0)} \prod_{i=1}^{N} f_{\delta_{N}}\left(v_{i}\right) \log f_{\delta_{N}}\left(v_{1}\right) d \sigma_{N, 0}^{N}-\log \left(Z_{B}^{N}\left(f_{\delta_{N}}, \sqrt{N}, 0\right)\right)
\end{gathered}
$$


Using Theorem 2.1 we find that

$$
\begin{aligned}
& \frac{1}{Z_{B}^{N}\left(f_{\delta_{N}}, \sqrt{N}, 0\right)} \int_{\mathscr{S}_{B}^{N}(N, 0)} \prod_{i=1}^{N} f_{\delta_{N}}\left(v_{i}\right) \log f_{\delta_{N}}\left(v_{1}\right) d \sigma_{N, 0}^{N}=\frac{\left|\mathbb{S}^{d(N-2)-1}\right|}{\left|\mathbb{S}^{d(N-1)-1}\right|} \cdot \frac{N^{\frac{d}{2}}}{(N-1)^{\frac{d}{2}}} \cdot \frac{1}{N^{\frac{d(N-1)-2}{2}}} \\
& \int_{\Pi_{1, N}} d v_{1}\left(N-\left|v_{1}\right|^{2}-\frac{\left|v_{1}\right|^{2}}{N-1}\right)^{\frac{d(N-2)-2}{2}} \cdot \frac{\mathcal{Z}_{N-1}\left(f_{\delta_{N}}, \sqrt{N-\left|v_{1}\right|^{2}},-v_{1}\right)}{Z_{N}\left(f_{\delta_{N}}, \sqrt{N}, 0\right)}
\end{aligned}
$$

At this point we notice that Theorem 3.4 can also be applied to $Z_{N-1}$ with the appropriate changes. This leads us to conclude that

$$
\begin{gathered}
\frac{\left|\mathbb{S}^{d(N-2)-1}\right|\left(N-\left|v_{1}\right|^{2}-\frac{\left|\nu_{1}\right|^{2}}{N-1}\right)^{\frac{d(N-2)-2}{2}}}{2(N-1)^{\frac{d}{2}}} Z_{N-1}\left(f_{\delta_{N}}, \sqrt{N-\left|v_{1}\right|^{2}},-v_{1}\right) \\
=\frac{d^{\frac{d}{2}}}{\sum_{\delta_{N}}^{2}(N-1)^{\frac{d+1}{2}}(2 \pi)^{\frac{d+1}{2}}}\left(e^{-\frac{d\left|\nu_{1}\right|^{2}}{2(N-1)}} e^{-\frac{\left(1-\left|\nu_{1}\right|^{2}\right)^{2}}{2 \Sigma_{\delta_{N}}^{2}(N-1)}}+\lambda\left(\sqrt{N-\left|v_{1}\right|^{2}},-v_{1}\right)\right)
\end{gathered}
$$

where $\sup _{v_{1} \in \Pi_{1, N}}\left|\lambda\left(\sqrt{N-\left|v_{1}\right|^{2},-v_{1}}\right)\right|=\epsilon_{1}(N) \underset{N \rightarrow \infty}{\longrightarrow} 0$.

Using Theorem 3.4 again we find that

$$
\frac{\left|\mathbb{S}^{d(N-1)-1}\right| N^{\frac{d(N-1)-2}{2}}}{2 N^{\frac{d}{2}}} Z_{N}\left(f_{\delta_{N}}, \sqrt{N}, 0\right)=\frac{d^{\frac{d}{2}}}{\sum_{\delta_{N}}^{2} N^{\frac{d+1}{2}}(2 \pi)^{\frac{d+1}{2}}}(1+\epsilon(N))
$$

where $\epsilon(N) \underset{N \rightarrow \infty}{\longrightarrow} 0$.

Combining equations (4.3) and (4.4) we find that

$$
\begin{gathered}
\frac{1}{Z_{B}^{N}\left(f_{\delta_{N}}, \sqrt{N}, 0\right)} \int_{\mathscr{S}_{B}^{N}(N, 0)} \prod_{i=1}^{N} f_{\delta_{N}}\left(v_{i}\right) \log f_{\delta_{N}}\left(\nu_{1}\right) d \sigma_{N, 0}^{N} \\
=\left(\frac{N}{N-1}\right)^{\frac{d+1}{2}} \int_{\Pi_{1, N}} \frac{e^{-\frac{d\left|v_{1}\right|^{2}}{2(N-1)}} e^{-\frac{\left(1-\left|\nu_{1}\right|^{2}\right)^{2}}{2 \Sigma_{\delta_{N}}^{2}(N-1)}}+\lambda\left(\sqrt{N-\left|v_{1}\right|^{2},-v_{1}}\right)}{1+\epsilon(N)} f_{\delta_{N}}\left(\nu_{1}\right) \log f_{\delta_{N}}\left(\nu_{1}\right) d v_{1}
\end{gathered}
$$

Rewriting $f_{\delta_{N}}(v)=d^{\frac{d}{2}}\left(\frac{\delta_{N}^{\frac{d+2}{2}}}{\pi^{\frac{d}{2}}} e^{-d \delta_{N}|\nu|^{2}}+\frac{\left(1-\delta_{N}\right)^{\frac{d+2}{2}}}{\pi^{\frac{d}{2}}} e^{-d\left(1-\delta_{N}\right)|\nu|^{2}}\right)=d^{\frac{d}{2}} f_{1, N}(v)$ we find that $0<f_{1, N}<1$ and as such

$$
\begin{aligned}
& \left|\chi_{\Pi_{1, N}}\left(\nu_{1}\right) \frac{e^{-\frac{d\left|v_{1}\right|^{2}}{2(N-1)}} e^{-\frac{\left(1-\left|\nu_{1}\right|^{2}\right)^{2}}{2 \Sigma_{\delta_{N}}^{2}(N-1)}}+\lambda\left(\sqrt{N-\left|v_{1}\right|^{2},-v_{1}}\right)}{1+\epsilon(N)} f_{\delta_{N}}\left(v_{1}\right) \log f_{\delta_{N}}\left(v_{1}\right)\right| \\
& \leq \frac{1+\left|\epsilon_{1}(N)\right|}{1-|\epsilon(N)|}\left(\frac{d \log d}{2} f_{\delta_{N}}\left(\nu_{1}\right)-f_{\delta_{N}}\left(\nu_{1}\right) \log f_{1, N}\left(\nu_{1}\right)\right) \\
& =\frac{1+\left|\epsilon_{1}(N)\right|}{1-|\epsilon(N)|}\left(d \log d \cdot f_{\delta_{N}}\left(v_{1}\right)-f_{\delta_{N}}\left(v_{1}\right) \log f_{\delta_{N}}\left(v_{1}\right)\right)
\end{aligned}
$$


A COUNTER EXAMPLE TO CERCIGNANI'S CONJECTURE FOR THE D DIMENSIONAL KAC MODEL - AMIT EIN\&V

$$
\begin{gathered}
\leq \frac{1+\left|\epsilon_{1}(N)\right|}{1-|\epsilon(N)|} \cdot d \log d \cdot f_{\delta_{N}}\left(\nu_{1}\right) \\
-\frac{1+\left|\epsilon_{1}(N)\right|}{1-|\epsilon(N)|}\left(\delta_{N} M_{\frac{1}{2 d \delta_{N}}}\left(v_{1}\right) \log \left(\delta_{N} M_{\frac{1}{2 d \delta_{N}}}\left(\nu_{1}\right)\right)+\left(1-\delta_{N}\right) M_{\frac{1}{2 d\left(1-\delta_{N}\right)}}\left(\nu_{1}\right) \log \left(\left(1-\delta_{N}\right) M_{\frac{1}{2 d\left(1-\delta_{N}\right)}}\left(\nu_{1}\right)\right)\right) \\
=\frac{1+\left|\epsilon_{1}(N)\right|}{1-|\epsilon(N)|} \cdot d \log d \cdot f_{\delta_{N}}\left(\nu_{1}\right) \\
+\frac{1+\left|\epsilon_{1}(N)\right|}{1-|\epsilon(N)|} \cdot \delta_{N} M_{\frac{1}{2 d \delta_{N}}}\left(\nu_{1}\right)\left(d \delta_{N}\left|v_{1}\right|^{2}-\frac{d}{2} \log \left(\frac{d}{\pi}\right)-\frac{d+2}{2} \log \left(\delta_{N}\right)\right) \\
\frac{1+\left|\epsilon_{1}(N)\right|}{1-|\epsilon(N)|} \cdot\left(1-\delta_{N}\right) M_{\frac{1}{2 d\left(1-\delta_{N}\right)}}\left(v_{1}\right)\left(d\left(1-\delta_{N}\right)\left|v_{1}\right|^{2}-\frac{d}{2} \log \left(\frac{d}{\pi}\right)-\frac{d+2}{2} \log \left(1-\delta_{N}\right)\right)=g_{N}\left(v_{1}\right)
\end{gathered}
$$

We notice that $g_{N}\left(v_{1}\right) \underset{N \rightarrow \infty}{\longrightarrow}\left(\frac{d \log d}{2}+\frac{d \log \pi}{2}+d\left|v_{1}\right|^{2}\right) M_{\frac{1}{2 d}}\left(v_{1}\right)$ and

$$
\begin{gathered}
\int_{\mathbb{R}^{d}} g_{N}\left(\nu_{1}\right) d \nu_{1}=\frac{1+\left|\epsilon_{1}(N)\right|}{1-|\epsilon(N)|}\left(d \log d+\frac{d \delta_{N}}{2}-\frac{d \delta_{N}}{2} \log \left(\frac{d}{\pi}\right)-\frac{(d+2) \delta_{N} \log \left(\delta_{N}\right)}{2}\right. \\
\left.+\frac{d\left(1-\delta_{N}\right)}{2}-\frac{d\left(1-\delta_{N}\right)}{2} \log \left(\frac{d}{\pi}\right)-\frac{(d+2)\left(1-\delta_{N}\right) \log \left(\left(1-\delta_{N}\right)\right.}{2}\right)
\end{gathered}
$$

Thus

$$
\lim _{N \rightarrow \infty} \int_{\mathbb{R}^{d}} g_{N}\left(v_{1}\right) d v_{1}=\frac{d \log d}{2}+\frac{d \log \pi}{2}+\frac{d}{2}=\int_{\mathbb{R}^{d}} \lim _{N \rightarrow \infty} g_{N}\left(v_{1}\right) d v_{1}
$$

Since clearly

$$
\begin{gathered}
\chi_{\Pi_{1, N}}\left(\nu_{1}\right) \frac{e^{-\frac{d\left|\nu_{1}\right|^{2}}{2(N-1)}} e^{-\frac{\left(1-\left|\nu_{1}\right|^{2}\right)^{2}}{2 \Sigma_{\delta_{N}}^{2}(N-1)}}+\lambda\left(\sqrt{N-\left|\nu_{1}\right|^{2},-v_{1}}\right)}{1+\epsilon(N)} f_{\delta_{N}}\left(\nu_{1}\right) \log f_{\delta_{N}}\left(\nu_{1}\right) \\
\underset{N \rightarrow \infty}{\longrightarrow} M_{\frac{1}{2 d}}\left(\nu_{1}\right) \log \left(M_{\frac{1}{2 d}}\left(\nu_{1}\right)\right)
\end{gathered}
$$

we conclude by the Generalised Dominated Convergence Theorem that

$$
\begin{gathered}
\lim _{N \rightarrow \infty} \frac{1}{\mathcal{Z}_{B}^{N}\left(f_{\delta_{N}}, \sqrt{N}, 0\right)} \int_{\mathscr{S}_{B}^{N}(N, 0)} \prod_{i=1}^{N} f_{\delta_{N}}\left(\nu_{i}\right) \log f_{\delta_{N}}\left(\nu_{1}\right) d \sigma_{N, 0}^{N} \\
\quad=\int_{\mathbb{R}^{d}} M_{\frac{1}{2 d}}(v) \log \left(M_{\frac{1}{2 d}}(v)\right) d v=\frac{d}{2} \log d-\frac{d}{2} \log \pi-\frac{d}{2}
\end{gathered}
$$

We're only left with the evaluation the term $\log \left(\mathfrak{Z}_{N}\left(f_{\delta_{N}, \sqrt{N}, 0}\right)\right)$ to complete the proof. Using (4.4) along with $\left|\mathbb{S}^{m-1}\right|=\frac{2 \pi^{\frac{m}{2}}}{\Gamma\left(\frac{m}{2}\right)}$ and an approximation for the gamma function yields the following:

$$
\begin{gathered}
Z_{N}\left(f_{\delta_{N}}, \sqrt{N}, 0\right)=\frac{2 d^{\frac{d}{2}}\left(1+\epsilon_{2}(N)\right)}{(2 \pi)^{\frac{d+1}{2}} \Sigma_{\delta_{N}^{2}} N^{\frac{d(N-1)-1}{2}}\left|S^{d(N-1)-1}\right|} \\
=\frac{d^{\frac{d}{2}} \pi^{-\frac{d N}{2}} \Gamma\left(\frac{d(N-1)}{2}\right)\left(1+\epsilon_{2}(N)\right)}{2^{\frac{d+1}{2}} \sqrt{\pi} \Sigma_{\delta_{N}^{2}} N^{\frac{d(N-1)-1}{2}}}
\end{gathered}
$$




$$
\begin{gathered}
=\frac{d^{\frac{d}{2}} \pi^{-\frac{d N}{2}}\left(\left(\frac{d(N-1)}{2}\right)^{\frac{d(N-1)-1}{2}} e^{-\frac{d(N-1)}{2}} \sqrt{2 \pi}\left(1+\epsilon_{3}(N)\right)\right)\left(1+\epsilon_{2}(N)\right)}{2^{\frac{d+1}{2}} \sqrt{\pi} \Sigma_{\delta_{N}^{2}} N^{\frac{d(N-1)-1}{2}}} \\
=\left(\frac{d e}{2}\right)^{\frac{d}{2}} \cdot \frac{(\pi e)^{-\frac{d N}{2}}}{\Sigma_{\delta_{N}}^{2}} \cdot\left(\frac{d}{2}\left(1-\frac{1}{N}\right)\right)^{\frac{d(N-1)-1}{2}} \cdot(1+\epsilon(N))
\end{gathered}
$$

Thus,

$$
\begin{gathered}
\lim _{N \rightarrow \infty} \frac{\log \left(Z_{N}\left(f_{\delta_{N}}, \sqrt{N}, 0\right)\right)}{N}=-\frac{d}{2} \log (\pi e)+\frac{d}{2} \log \left(\frac{d}{2}\right) \\
=\frac{d \log d}{2}-\frac{d \log \pi}{2}-\frac{d}{2}-\frac{d \log 2}{2}
\end{gathered}
$$

Combining (4.2), (4.5) and (4.6) yields the result.

Theorem 4.2. There exists a constant $C_{\delta}$, depending only on the behaviour of $\delta$ such that

$$
\left\langle\log F_{N},(I-Q) F_{N}\right\rangle \leq-C_{\delta} \delta_{N} \log \delta_{N}
$$

Proof. Since $\left\langle C,(I-Q) F_{N}\right\rangle=0$ for any constant $C$, and with the same notation of the proof of Theorem 4.2, we find that

$$
\begin{gathered}
\left\langle\log F_{N},(I-Q) F_{N}\right\rangle=\frac{2}{\left.N(N-1) \mathfrak{Z}_{N}\left(f_{\delta_{N}}, \sqrt{N}, 0\right)\right)} \sum_{i<j} \int_{\mathscr{S}_{B}^{N}(N, 0)} d \sigma_{N, 0}^{N} \log \left(\prod_{k=1}^{N} f_{1, N}\left(v_{k}\right)\right) \\
\int_{\mathbb{S}^{d-1}}\left[f_{\delta_{N}}^{\otimes N}\left(v_{1}, \ldots, v_{i}, \ldots, v_{j}, \ldots, v_{N}\right)-f_{\delta_{N}}^{\otimes N}\left(v_{1}, \ldots, v_{i}(\omega), \ldots, v_{j}(\omega), \ldots, v_{N}\right)\right] d \omega \\
=\frac{2}{N(N-1) \mathfrak{Z}_{N}\left(f_{\delta_{N}}, \sqrt{N}, 0\right)} \sum_{i<j} \sum_{k=1}^{N} \int_{\mathscr{S}_{B}^{N}(N, 0)} d \sigma_{N, 0}^{N} \log \left(f_{1, N}\left(v_{k}\right)\right) \\
\int_{\mathbb{S}^{d-1}}\left[f_{\delta_{N}}^{\otimes N}\left(v_{1}, \ldots, v_{i}, \ldots, v_{j}, \ldots, v_{N}\right)-f_{\delta_{N}}^{\otimes N}\left(v_{1}, \ldots, v_{i}(\omega), \ldots, v_{j}(\omega), \ldots, v_{N}\right)\right] d \omega
\end{gathered}
$$

We notice that if $k \neq i, j$ then the integral is equals to

$$
\begin{gathered}
\frac{\left|\mathbb{S}^{d(N-2)-1}\right|}{\left|\mathbb{S}^{d(N-1)-1}\right|} \cdot \frac{N^{\frac{d}{2}}}{(N-1)^{\frac{d}{2}} N^{\frac{d(N-1)-2}{2}}} \int_{\mathbb{S}^{d-1}} d \omega \int_{v_{k} \in \Pi_{1, N}} \log \left(f_{1, N}\left(v_{k}\right)\right)\left(N-\left|v_{k}\right|^{2}-\frac{\left|v_{k}\right|^{2}}{N-1}\right)^{\frac{d(N-2)-2}{2}} \\
\int_{\mathscr{S}_{B}^{N-1}\left(N-\left|v_{k}\right|^{2},-v_{k}\right)}\left[f_{\delta_{N}}^{\otimes N}\left(v_{1}, \ldots, v_{i}, \ldots, v_{j}, \ldots, v_{N}\right)-f_{\delta_{N}}^{\otimes N}\left(v_{1}, \ldots, v_{i}(\omega), \ldots, v_{j}(\omega), \ldots, v_{N}\right)\right] d \sigma_{N-\left|v_{k}\right|^{2},-v_{k}}^{N-1} \\
=0
\end{gathered}
$$

due to the invariance of the Boltzmann sphere under the map $\left(v_{i}, v_{j}\right) \rightarrow\left(v_{i}(\omega), v_{j}(\omega)\right)$. Also, since the above map is invariant under switching the $i$-th and $j$-th indexes, we can have that

$$
\begin{gathered}
\left\langle\log F_{N},(I-Q) F_{N}\right\rangle=\frac{2}{N(N-1) \mathcal{Z}_{N}\left(f_{\delta_{N}}, \sqrt{N}, 0\right)} \sum_{i<j} \int_{\mathscr{S}_{B}^{N}(N, 0)} d \sigma_{N, 0}^{N}\left(\log \left(f_{1, N}\left(v_{i}\right)\right)+\log \left(f_{1, N}\left(v_{j}\right)\right)\right) \\
\int_{\mathbb{S}^{d-1}}\left[f_{\delta_{N}}^{\otimes N}\left(v_{1}, \ldots, v_{i}, \ldots, v_{j}, \ldots, v_{N}\right)-f_{\delta_{N}}^{\otimes N}\left(v_{1}, \ldots, v_{i}(\omega), \ldots, v_{j}(\omega), \ldots, v_{N}\right)\right] d \omega
\end{gathered}
$$


A COUNTER EXAMPLE TO CERCIGNANI'S CONJECTURE FOR THE D DIMENSIONAL KAC MODEL - AMIT EINZY

$$
\begin{aligned}
& =\frac{2}{N(N-1) Z_{N}\left(f_{\delta_{N}}, \sqrt{N}, 0\right)} \sum_{i \neq j} \int_{\mathscr{S}_{B}^{N}(N, 0)} d \sigma_{N, 0}^{N} \log \left(f_{1, N}\left(v_{i}\right)\right) \\
& \int_{\mathbb{S}^{d-1}}\left[f_{\delta_{N}}^{\otimes N}\left(v_{1}, \ldots, v_{i}, \ldots, v_{j}, \ldots, v_{N}\right)-f_{\delta_{N}}^{\otimes N}\left(v_{1}, \ldots, v_{i}(\omega), \ldots, v_{j}(\omega), \ldots, v_{N}\right)\right] d \omega \\
& =\frac{2}{Z_{N}\left(f_{\delta_{N}}, \sqrt{N}, 0\right)} \\
& \int_{\mathscr{S}_{B}^{N}(N, 0)} d \sigma_{N, 0}^{N} \log \left(f_{1, N}\left(v_{1}\right)\right) \int_{\mathbb{S}^{d-1}}\left[f_{\delta_{N}}^{\otimes N}\left(v_{1}, v_{2}, \ldots, v_{N}\right)-f_{\delta_{N}}^{\otimes N}\left(v_{1}(\omega), v_{2}(\omega), \ldots, v_{N}\right)\right] d \omega \\
& =\frac{2}{Z_{N}\left(f_{\delta_{N}}, \sqrt{N}, 0\right)} \int_{\mathbb{S}^{d-1}} d \omega \frac{\left|\mathbb{S}^{d(N-3)-1}\right|}{\left|\mathbb{S}^{d(N-1)-1}\right|} \cdot \frac{N^{\frac{d}{2}}}{(N-2)^{\frac{d}{2}} N^{\frac{d(N-1)-2}{2}}} \\
& \int_{\Pi_{2, N}} d v_{1} d v_{2}\left(N-\left(\left|v_{1}\right|^{2}+\left|v_{2}\right|^{2}\right)-\frac{\left|v_{1}+v_{2}\right|^{2}}{N-2}\right)^{\frac{d(N-3)-2}{2}} \log \left(f_{1, N}\left(v_{1}\right)\right) \\
& \left(f_{\delta_{N}}\left(\nu_{1}\right) f_{\delta_{N}}\left(v_{2}\right)-f_{\delta_{N}}\left(v_{1}(\omega)\right) f_{\delta_{N}}\left(v_{2}(\omega)\right)\right) Z_{N-2}\left(f_{\delta_{N}}, \sqrt{N-\left(\left|v_{1}\right|^{2}+\left|v_{2}\right|^{2}\right)},-v_{1}-v_{2}\right)
\end{aligned}
$$

Using Theorem 3.4 for $\mathfrak{Z}_{N-2}$ (with the appropriate changes) gives us

$$
\begin{gathered}
\frac{\left|\mathbb{S}^{d(N-3)-1}\right|\left(N-\left(\left|v_{1}\right|^{2}+\left|v_{2}\right|^{2}\right)-\frac{\left|v_{1}+\nu_{2}\right|^{2}}{N-2}\right)^{\frac{d(N-3)-2}{2}}}{2(N-2)^{\frac{d}{2}}} Z_{N-2}\left(f_{\delta_{N}}, \sqrt{N-\left(\left|v_{1}\right|^{2}+\left|v_{2}\right|^{2}\right)},-v_{1}-v_{2}\right) \\
=\frac{d^{\frac{d}{2}}}{\sum_{\delta_{N}}^{2}(N-2)^{\frac{d+1}{2}}(2 \pi)^{\frac{d+1}{2}}}\left(e^{-\frac{d\left|\nu_{1}+\nu_{2}\right|^{2}}{2(N-2)}} e^{-\frac{\left(2-\left|\nu_{1}\right|^{2}-\left|v_{2}\right|^{2}\right)^{2}}{2 \Sigma_{\delta_{N}}(N-2)}}+\lambda\left(\sqrt{N-\left(\left|v_{1}\right|^{2}+\left|v_{2}\right|^{2}\right)},-v_{1}-v_{2}\right)\right)
\end{gathered}
$$

where $\sup _{v_{1}, \nu_{2} \in \Pi_{2, N}}\left|\lambda\left(\sqrt{N-\left(\left|v_{1}\right|^{2}+\left|v_{2}\right|^{2}\right)},-v_{1}-v_{2}\right)\right|=\epsilon_{1}(N) \underset{N \rightarrow \infty}{\longrightarrow} 0$.

Plugging (4.8) and (4.4) into our equation we find that

$$
\begin{aligned}
& \left\langle\log F_{N},(I-Q) F_{N}\right\rangle=2\left(\frac{N}{N-2}\right)^{\frac{d+1}{2}} \int_{\mathbb{S}^{d-1}} \int_{\Pi_{2, N}} d v_{1} d v_{2} d \omega \\
& \frac{e^{-\frac{d\left|\nu_{1}+\nu_{2}\right|^{2}}{2(N-2)}} e^{-\frac{\left(2-\left|\nu_{1}\right|^{2}-\left|\nu_{2}\right|^{2}\right)^{2}}{2 \Sigma_{\delta_{N}}^{2}(N-2)}}+\lambda\left(\sqrt{N-\left(\left|\nu_{1}\right|^{2}+\left|v_{2}\right|^{2}\right)},-v_{1}-v_{2}\right)}{1+\epsilon(N))} \\
& \log \left(f_{1, N}\left(v_{1}\right)\right)\left(f_{\delta_{N}}\left(\nu_{1}\right) f_{\delta_{N}}\left(v_{2}\right)-f_{\delta_{N}}\left(\nu_{1}(\omega)\right) f_{\delta_{N}}\left(v_{2}(\omega)\right)\right)
\end{aligned}
$$

at this point we notice that since $\left|v_{1}\right|^{2}+\left|v_{2}\right|^{2}=\left|v_{1}(\omega)\right|^{2}+\left|v_{2}(\omega)\right|^{2}$ and $v_{1}+v_{2}=$ $v_{1}(\omega)+v_{2}(\omega)$ the domain $\Pi_{2, N}$ is symmetric to changing 1 with 2 and $v$ with $v(\omega)$.

Thus we can rewrite the above as

$$
\begin{gathered}
\left\langle\log F_{N},(I-Q) F_{N}\right\rangle=\frac{1}{2}\left(\frac{N}{N-2}\right)^{\frac{d+1}{2}} \int_{\mathbb{S}^{d-1}} \int_{\Pi_{2, N}} d v_{1} d v_{2} d \omega \\
\frac{e^{-\frac{d\left|\nu_{1}+v_{2}\right|^{2}}{2(N-2)}} e^{-\frac{\left(2-\left|\nu_{1}\right|^{2}-\left|v_{2}\right|^{2}\right)^{2}}{2 \Sigma_{\delta_{N}}^{2}(N-2)}}+\lambda\left(\sqrt{N-\left(\left|\nu_{1}\right|^{2}+\left|v_{2}\right|^{2}\right)},-v_{1}-v_{2}\right)}{1+\epsilon(N))}
\end{gathered}
$$




$$
\log \left(\frac{f_{1, N}\left(\nu_{1}\right) f_{1, N}\left(v_{2}\right)}{f_{1, N}\left(\nu_{1}(\omega)\right) f_{1, N}\left(v_{2}(\omega)\right)}\right)\left(f_{\delta_{N}}\left(\nu_{1}\right) f_{\delta_{N}}\left(\nu_{2}\right)-f_{\delta_{N}}\left(\nu_{1}(\omega)\right) f_{\delta_{N}}\left(v_{2}(\omega)\right)\right)
$$

whose integrand is clearly non-negative. As such

$$
\begin{aligned}
& \left\langle\log F_{N},(I-Q) F_{N}\right\rangle \leq \frac{1}{2}\left(\frac{N}{N-2}\right)^{\frac{d+1}{2}} \int_{\mathbb{S}^{d-1}} \int_{\mathbb{R}^{2 d}} d v_{1} d v_{2} d \omega \\
& \frac{e^{-\frac{d\left|\nu_{1}+v_{2}\right|^{2}}{2(N-2)}} e^{-\frac{\left(2-\left|v_{1}\right|^{2}-\left|\nu_{2}\right|^{2}\right)^{2}}{2 \Sigma_{\delta_{N}}^{2}(N-2)}}+\lambda\left(\sqrt{N-\left(\left|v_{1}\right|^{2}+\left|v_{2}\right|^{2}\right)},-v_{1}-v_{2}\right)}{1+\epsilon(N))} \\
& \log \left(\frac{f_{1, N}\left(\nu_{1}\right) f_{1, N}\left(v_{2}\right)}{f_{1, N}\left(v_{1}(\omega)\right) f_{1, N}\left(\nu_{2}(\omega)\right)}\right)\left(f_{\delta_{N}}\left(\nu_{1}\right) f_{\delta_{N}}\left(\nu_{2}\right)-f_{\delta_{N}}\left(\nu_{1}(\omega)\right) f_{\delta_{N}}\left(\nu_{2}(\omega)\right)\right) \\
& =2\left(\frac{N}{N-2}\right)^{\frac{d+1}{2}} \int_{\mathbb{S}^{d-1}} \int_{\mathbb{R}^{2 d}} d v_{1} d v_{2} d \omega \\
& \frac{e^{-\frac{d\left|\nu_{1}+v_{2}\right|^{2}}{2(N-2)}} e^{-\frac{\left(2-\left|v_{1}\right|^{2}-\left|v_{2}\right|^{2}\right)^{2}}{2 \Sigma_{\delta_{N}}^{2}(N-2)}}+\lambda\left(\sqrt{N-\left(\left|v_{1}\right|^{2}+\left|v_{2}\right|^{2}\right)},-v_{1}-v_{2}\right)}{1+\epsilon(N))} \\
& \log \left(f_{1, N}\left(\nu_{1}\right)\right)\left(f_{\delta_{N}}\left(\nu_{1}\right) f_{\delta_{N}}\left(\nu_{2}\right)-f_{\delta_{N}}\left(\nu_{1}(\omega)\right) f_{\delta_{N}}\left(v_{2}(\omega)\right)\right) \\
& \leq \frac{2\left(1+\mid \epsilon_{1}(N)\right) \mid}{1-\mid \epsilon(N)) \mid}\left(\frac{N}{N-2}\right)^{\frac{d+1}{2}} \int_{\mathbb{S}^{d-1}} \int_{\mathbb{R}^{2 d}} d v_{1} d v_{2} d \omega \\
& \left|\log \left(f_{1, N}\left(v_{1}\right)\right)\right|\left|\left(f_{\delta_{N}}\left(v_{1}\right) f_{\delta_{N}}\left(v_{2}\right)-f_{\delta_{N}}\left(\nu_{1}(\omega)\right) f_{\delta_{N}}\left(v_{2}(\omega)\right)\right)\right|
\end{aligned}
$$

and since $0<f_{1, N}<1$ we conclude that

$$
\begin{gathered}
\left\langle\log F_{N},(I-Q) F_{N}\right\rangle \leq \frac{2\left(1+\mid \epsilon_{1}(N)\right) \mid}{1-\mid \epsilon(N)) \mid}\left(\frac{N}{N-2}\right)^{\frac{d+1}{2}} \int_{\mathbb{S}^{d-1}} \int_{\mathbb{R}^{2 d}} d v_{1} d v_{2} d \omega \\
\left(-\log \left(f_{1, N}\left(v_{1}\right)\right)\right)\left|\left(f_{\delta_{N}}\left(\nu_{1}\right) f_{\delta_{N}}\left(\nu_{2}\right)-f_{\delta_{N}}\left(\nu_{1}(\omega)\right) f_{\delta_{N}}\left(\nu_{2}(\omega)\right)\right)\right|
\end{gathered}
$$

Next, we notice that

$$
\begin{gathered}
-\log \left(f_{1, N}\left(\nu_{1}\right)\right) \leq-\log \left(\frac{\delta_{N}^{\frac{d+2}{2}}}{\pi^{\frac{d}{2}}} e^{-d \delta_{N}\left|\nu_{1}\right|^{2}}\right) \\
\leq d \delta_{N}\left(\left|v_{1}\right|^{2}+\left|v_{2}\right|^{2}\right)+\frac{d \log \pi}{2}-\frac{d+2}{2} \log \left(\delta_{N}\right)
\end{gathered}
$$

Also, since

$$
\begin{gathered}
f_{\delta_{N}}\left(\nu_{1}\right) f_{\delta_{N}}\left(v_{2}\right)=\delta_{N}^{2} M_{\frac{1}{2 d \delta_{N}}}\left(\nu_{1}\right) M_{\frac{1}{2 d \delta_{N}}}\left(v_{2}\right) \\
+\delta_{N}\left(1-\delta_{N}\right)\left(M_{\frac{1}{2 d \delta_{N}}}\left(\nu_{1}\right) M_{\frac{1}{2 d\left(1-\delta_{N}\right)}}\left(\nu_{2}\right)+M_{\frac{1}{2 d\left(1-\delta_{N}\right)}}\left(v_{1}\right) M_{\frac{1}{2 d \delta_{N}}}\left(v_{2}\right)\right) \\
\left(1-\delta_{N}\right)^{2} M_{\frac{1}{2 d\left(1-\delta_{N}\right)}}\left(\nu_{1}\right) M_{\frac{1}{2 d\left(1-\delta_{N}\right)}}\left(v_{2}\right)
\end{gathered}
$$


We find that

$$
\begin{gathered}
\left|\left(f_{\delta_{N}}\left(\nu_{1}\right) f_{\delta_{N}}\left(\nu_{2}\right)-f_{\delta_{N}}\left(\nu_{1}(\omega)\right) f_{\delta_{N}}\left(\nu_{2}(\omega)\right)\right)\right| \\
\leq \delta_{N}\left(1-\delta_{N}\right)\left(M_{\frac{1}{2 d \delta_{N}}}\left(\nu_{1}\right) M_{\frac{1}{2 d\left(1-\delta_{N}\right)}}\left(\nu_{2}\right)+M_{\frac{1}{2 d\left(1-\delta_{N}\right)}}\left(\nu_{1}\right) M_{\frac{1}{2 d \delta_{N}}}\left(\nu_{2}\right)\right. \\
\left.+M_{\frac{1}{2 d \delta_{N}}}\left(\nu_{1}(\omega)\right) M_{\frac{1}{2 d\left(1-\delta_{N}\right)}}\left(\nu_{2}(\omega)\right)+M_{\frac{1}{2 d\left(1-\delta_{N}\right)}}\left(\nu_{1}(\omega)\right) M_{\frac{1}{2 d \delta_{N}}}\left(\nu_{2}(\omega)\right)\right)
\end{gathered}
$$

Plugging (4.10) and (4.11) into (4.9) and using symmetry we find that

$$
\begin{gathered}
\left\langle\log F_{N},(I-Q) F_{N}\right\rangle \leq \frac{8\left(1+\mid \epsilon_{1}(N)\right) \mid}{1-\mid \epsilon(N)) \mid}\left(\frac{N}{N-2}\right)^{\frac{d+1}{2}} \delta_{N}\left(1-\delta_{N}\right) \\
\int_{\mathbb{R}^{2 d}}\left(d \delta_{N}\left(\left|\nu_{1}\right|^{2}+\left|v_{2}\right|^{2}\right)+\frac{d \log \pi}{2}-\frac{d+2}{2} \log \left(\delta_{N}\right)\right) M_{\frac{1}{2 d \delta_{N}}}\left(\nu_{1}\right) M_{\frac{1}{2 d\left(1-\delta_{N}\right)}}\left(\nu_{2}\right) d \nu_{1} d \nu_{2} \\
=\frac{8\left(1+\mid \epsilon_{1}(N)\right) \mid}{1-\mid \epsilon(N)) \mid}\left(\frac{N}{N-2}\right)^{\frac{d+1}{2}} \delta_{N}\left(1-\delta_{N}\right)\left(\frac{d \log \pi}{2}+\frac{d}{2\left(1-\delta_{N}\right)}-\frac{d+2}{2} \log \delta_{N}\right)
\end{gathered}
$$

which proves the result.

Proof of Theorem 1.1. With the same family of functions as in Theorems 4.1 and 4.2 we find that

$$
\Gamma_{N} \leq \frac{\left\langle\log F_{N}, N(I-Q) F_{N}\right\rangle}{H_{N}\left(F_{N}\right)}=\frac{\left\langle\log F_{N},(I-Q) F_{N}\right\rangle}{\frac{H\left(F_{N}\right)}{N}} \leq-C_{\delta} \delta_{N} \log \delta_{N}
$$

and plugging $\delta_{N}=\frac{1}{N^{1-\eta}}$, with $\eta$ satisfying the conditions of Theorem 3.4 for an arbitrary $\beta>0$, yields the result.

\section{FinAL REMARKS}

In this paper we managed to see that the addition of higher dimension, allowing conservation of momentum as well as energy, doesn't help the entropyentropy production ratio. Nor does it worsen it. Questions that were raised in the one dimensional case are still valid here:

- For our specific choice of 'generating function', $f_{\delta_{N}}$, we notice that the fourth moment, connected to $\Sigma_{\delta_{N}}^{2}$, explodes as $N$ goes to infinity. Would restricting such behaviour result in a better ratio?

- Intuitively speaking, a reason for such 'slow relaxation' lies in the fact that we're trying to equilibrate many 'stable' states (represented by the Maxwellian with parameter $\left.\frac{1}{2\left(1-\delta_{N}\right)}\right)$ with very few highly energetic states (represented by the Maxwellian with parameter $\frac{1}{2 \delta_{N}}$ ). Will restricting our class of function to one where the velocities are 'close' in some sense result in a better ratio?

Another question that can be asked in the multi dimensional case is the following:

- Can one extend Villani's proof in [12] to the $d$-dimensional case? 
While we have no answers to any of the above so far, we're hoping that some of the presented questions will be solved, for the one dimensional case as well as for $d$-dimensions.

\section{Appendix A. Additional Proofs}

This Appendix contains several proofs of Lemmas that would have encumbered the main article, but pose a necessary step in the proof of our main result.

\section{Theorem A.1.}

$$
\begin{gathered}
\int_{\mathscr{S}_{B}^{N}(E, z)} F d \sigma_{E, z}^{N}=\frac{\left|\mathbb{S}^{d(N-j-1)-1}\right|}{\left|\mathbb{S}^{d(N-1)-1}\right|} \cdot \frac{N^{\frac{d}{2}}}{(N-j)^{\frac{d}{2}}\left(E-\frac{|z|^{2}}{N}\right)^{\frac{d(N-1)-2}{2}}} \\
\int_{\Pi_{j}(E, z)} d v_{1} \ldots d v_{j}\left(E-\sum_{i=1}^{j}\left|v_{i}\right|^{2}-\frac{\left|z-\sum_{i=1}^{j} v_{i}\right|^{2}}{N-j}\right)^{\frac{d(N-j-1)-2}{2}} \\
\int_{\mathscr{S}_{B}^{N-j}\left(E-\sum_{i=i}^{j}\left|v_{i}\right|^{2}, z-\sum_{i=1}^{j} \nu_{i}\right)} F d \sigma_{E-\sum_{i=i}^{j}\left|\nu_{i}\right|^{2}, z-\sum_{i=1}^{j} v_{i}}^{N-j}
\end{gathered}
$$

where $\Pi_{j}(E, z)=\left\{\sum_{i=1}^{j}\left|v_{i}\right|^{2}+\frac{\left|z-\sum_{i=1}^{j} v_{i}\right|^{2}}{N-j} \leq E\right\}$

Proof. The proof relies heavily on the transformation (2.1) and the following Fubini-like formula for spheres (which can be found in [6]):

$$
\begin{gathered}
\int_{\mathbb{S}^{m-1}(r)} f d \gamma_{r}^{m}=\frac{\left|\mathbb{S}^{m-j-1}\right|}{\left|\mathbb{S}^{m-1}\right| r^{m-2}} \\
\int_{\sum_{i=1}^{j} x_{i}^{2} \leq r^{2}} d x_{1} \ldots d x_{j}\left(r^{2}-\sum_{i=1}^{j} x_{i}^{2}\right)^{\frac{m-j-2}{2}} \int_{\mathbb{S}^{m-j-1}\left(\sqrt{r^{2}-\sum_{i=1}^{j} x_{i}^{2}}\right)} f d \gamma^{m-j} \sqrt{r^{2}-\sum_{i=1}^{j} x_{i}^{2}}
\end{gathered}
$$

where $d \gamma_{r}^{m}$ is the uniform probability measure on the appropriate sphere.

We start by defining the new variables

$$
\begin{aligned}
\left(\xi_{1}, \ldots, \xi_{j}\right) & =R_{1}\left(v_{1}, \ldots, v_{j}\right) \\
\left(\xi_{j+1}, \ldots, \xi_{N}\right) & =R_{2}\left(v_{j+1}, \ldots, v_{N}\right)
\end{aligned}
$$

where $R_{1}, R_{2}$ are transformation like (2.1). We notice that under the above transformation the domain

transforms into

$$
\sum_{i=1}^{N}\left|v_{i}\right|^{2}=E \quad \sum_{i=1}^{N} v_{i}=z
$$

which can be written as

$$
\sum_{i=1}^{N}\left|\xi_{i}\right|^{2}=E \quad \sqrt{j} \xi_{j}+\sqrt{N-j} \xi_{N}=z
$$

$$
\sum_{i=1}^{N-1}\left|\xi_{i}\right|^{2}+\frac{1}{N-j}\left|z-\sqrt{j} \xi_{j}\right|^{2}=E
$$



the next easy computation

$$
\begin{gathered}
x^{2}+\frac{1}{N-j}(z-\sqrt{j} x)^{2}=x^{2}+\frac{1}{N-j}\left(|z|^{2}-2 \sqrt{j} z x+j x^{2}\right) \\
=\frac{1}{N-j}\left(|z|^{2}-2 \sqrt{j} z x+N x^{2}\right)=\frac{1}{N-j}\left(N\left(x-\frac{\sqrt{j} z}{N}\right)^{2}+\frac{(N-j)|z|^{2}}{N}\right)
\end{gathered}
$$

shows that (A.2) is

$$
\sum_{i=1, i \neq j}^{N-1}\left|\xi_{i}\right|^{2}+\frac{N}{N-j}\left(\xi_{j}-\frac{\sqrt{j} z}{N}\right)^{2}=E-\frac{|z|^{2}}{N}
$$

denoting by $\widetilde{\xi}_{j}=\sqrt{\frac{N}{N-j}}\left(\xi_{j}-\frac{\sqrt{j} z}{N}\right)$ and using the fact that $R$ is orthogonal along with (A.1) we find that

$$
\begin{aligned}
& \int_{\mathscr{S}_{B}^{N}(E, z)} f d \sigma_{E, z}^{N}=\int_{\sum_{i=1, i \neq j}^{N-1}\left|\xi_{i}\right|^{2}+\left|\widetilde{\xi}_{j}\right|^{2}=E-\frac{|z|^{2}}{N}} f \circ R^{T} d \gamma_{\sqrt{E-\frac{|z|^{2}}{N}}}^{N(d-1)} \\
& =\frac{\left|\mathbb{S}^{d(N-1)-j-1}\right|}{\left|\mathbb{S}^{d(N-1)-1}\right|\left(E-\frac{|z|^{2}}{N}\right)^{\frac{d(N-1)-2}{2}}} \\
& \int_{\sum_{i=1}^{j-1}\left|\xi_{i}\right|^{2}+\left|\widetilde{\xi}_{j}\right|^{2} \leq E-\frac{\left.z\right|^{2}}{N}} d \xi_{1} \ldots d \xi_{j-1} d \widetilde{\xi}_{j}\left(E-\frac{|z|^{2}}{N}-\sum_{i=1}^{j-1}\left|\xi_{i}\right|^{2}-\left|\widetilde{\xi}_{j}\right|^{2}\right)^{\frac{d(N-j-1)-2}{2}} \\
& \int_{\mathbb{S}^{d(N-j-1)}\left(\sqrt{E-\frac{\left.|z|\right|^{2}}{N}-\sum_{i=1}^{j-1}\left|\xi_{i}\right|^{2}-\left|\widetilde{\xi}_{j}\right|^{2}}\right)} f \circ R^{T} d \gamma^{d(N-j)} \sqrt{E-\frac{|z|^{2}}{N}-\sum_{i=1}^{j-1}\left|\xi_{i}\right|^{2}-\left|\widetilde{\xi_{j}}\right|^{2}}
\end{aligned}
$$

Since

we find that

$$
\begin{gathered}
E-\frac{|z|^{2}}{N}-\sum_{i=1}^{j-1}\left|\xi_{i}\right|^{2}-\left|\widetilde{\xi}_{j}\right|^{2}=E-\sum_{i=1}^{j}\left|\xi_{i}\right|^{2}-\frac{\left|z-\sqrt{j} \xi_{j}\right|^{2}}{N-j} \\
=E-\sum_{i=1}^{j}\left|v_{i}\right|^{2}-\frac{\left|z-\sum_{i=1}^{j} v_{i}\right|^{2}}{N-j}
\end{gathered}
$$

$$
\begin{gathered}
\int_{\mathscr{S}_{B}^{N}(E, z)} f d \sigma_{E, z}^{N}=\frac{\left|\mathbb{S}^{d(N-1)-j-1}\right|}{\left|\mathbb{S}^{d(N-1)-1}\right|\left(E-\frac{|z|^{2}}{N}\right)^{\frac{d(N-1)-2}{2}}} \cdot\left(\frac{N}{N-j}\right)^{\frac{d}{2}} \\
\int_{\sum_{i=1}^{j}\left|v_{i}\right|^{2}+\frac{\left|z-\sum_{i=1}^{j} v_{i}\right|^{2}}{N-j} \leq E} d v_{1} \ldots d v_{j}\left(E-\sum_{i=1}^{j}\left|v_{i}\right|^{2}-\frac{\left|z-\sum_{i=1}^{j} v_{i}\right|^{2}}{N-j}\right)^{\frac{d(N-j-1)-2}{2}} \\
\int_{\mathscr{S}_{B}^{N-j}\left(E-\sum_{i=i}^{j}\left|v_{i}\right|^{2}, z-\sum_{i=1}^{j} v_{i}\right)} f d \sigma_{E-\sum_{i=i}^{N-j}\left|v_{i}\right|^{2}, z-\sum_{i=1}^{j} v_{i}}^{N}
\end{gathered}
$$

Lemma A.2. The function ${\widehat{h_{\delta}}}^{n}$ defined in (3.2) belongs to $L^{q}\left(\mathbb{R}^{d+1}\right)$ for any $n>$ $\frac{2(1+d)}{q d}$. 
Proof. By the definition, it is sufficient to show that ${\widehat{h_{a}}}^{j}{\widehat{h_{b}}}^{n-j}$ is in $L^{q}\left(\mathbb{R}^{d+1}\right)$ for all $j=0,1, \ldots, n$ and $a, b>0$ ( $\widehat{h_{a}}$ was defined in the proof of Lemma 3.2). Indeed

$$
\begin{aligned}
& \int_{\mathbb{R}^{d+1}}\left|\widehat{h_{a}}(p, t)\right|^{j q}\left|\widehat{h_{b}}(p, t)\right|^{(n-j) q} d p d t \\
= & \int_{\mathbb{R}^{d+1}} \frac{e^{-|p|^{2}\left(\frac{2 a q j \pi^{2}}{1+16 \pi^{2} a^{2} t^{2}}+\frac{2 b q(n-j) \pi^{2}}{1+16 \pi^{2} a^{2} t^{2}}\right)}}{\left(1+16 \pi^{2} a^{2} t^{2}\right)^{\frac{d q j}{4}}\left(1+16 \pi^{2} b^{2} t^{2}\right)^{\frac{d q(n-j)}{4}}} d p d t \\
= & C_{d} \int_{\mathbb{R}} \frac{\left(\frac{2 a q j \pi^{2}}{1+16 \pi^{2} a^{2} t^{2}}+\frac{2 b q(n-j) \pi^{2}}{1+16 \pi^{2} b^{2} t^{2}}\right)^{-\frac{d}{2}}}{\left(1+16 \pi^{2} a^{2} t^{2}\right)^{\frac{d q j}{4}}\left(1+16 \pi^{2} b^{2} t^{2}\right)^{\frac{d q(n-j)}{4}}} d p d t
\end{aligned}
$$

where $C_{d}=\int_{\mathbb{R}^{d}} e^{-|x|^{2}} d x$.

The behaviour at infinity is that of $\frac{1}{t^{d\left(\frac{n q}{2}-1\right)}}$ and thus we conclude that ${\widehat{h_{\delta}}}^{n} \in$ $L^{q}\left(\mathbb{R}^{d} \times[0, \infty)\right)$ for any $n>\frac{2(1+d)}{q d}$

Lemma A.3. Let $F(x)$ be a continuous function in $L^{q}\left(\mathbb{R}^{d+1}\right)$ for some $q>1$ and let $P$ be a probability measure such that for any $\varphi \in C_{c}\left(\mathbb{R}^{d+1}\right)$ we have

$$
\int_{\mathbb{R}^{d+1}} \varphi(x) F(x) d x=\int_{\mathbb{R}^{d+1}} \varphi(x) d P(x)
$$

Then $F \geq 0, F(x) \in L^{1}\left(\mathbb{R}^{d+1}\right)$ and $d P(x)=F(x) d x$.

Proof. Let $E$ be any bounded Borel set. Given an $\epsilon>0$ we can find open sets $U_{1}, U_{2}$ and compact sets $C_{1}, C_{2}$ such that $C_{i} \subset E \subset U_{i}$ for $i=1,2$ and $P\left(U_{1} \backslash C_{1}\right)<$ $\epsilon$ and $\lambda\left(U_{2} \backslash C_{2}\right)<\epsilon$ where $\lambda$ represents the Lebesgue measure. Defining $U=$ $U_{1} \cap U_{2}$ and $C=C_{1} \cup C_{2}$ we find an open and compact sets, bounding $E$ between them, such that $P(U \backslash C)<\epsilon$ and $\lambda(U \backslash C)<\epsilon$.

By Uryson's lemma we can find a function $\varphi_{\epsilon} \in C_{c}\left(\mathbb{R}^{d+1}\right)$ such that $0 \leq \varphi_{\epsilon} \leq 1$, $\left.\varphi_{\epsilon}\right|_{C}=1$ and $\left.\varphi_{\epsilon}\right|_{U^{c}}=0$

We have that

$$
\begin{aligned}
& \int_{\mathbb{R}^{d+1}}\left|\chi_{E}-\varphi_{\epsilon}\right||F(x)| d x=\int_{U \backslash C}\left|\chi_{E}-\varphi_{\epsilon}\right||F(x)| d x \\
\leq & \left(\int_{U \backslash C} d x\right)^{\frac{1}{q^{*}}} \cdot\left(\int_{U \backslash C}|F(x)|^{q} d x\right)^{\frac{1}{q}} \leq \sqrt[q^{*}]{\epsilon} \cdot\|F\|_{L^{q}\left(\mathbb{R}^{d+1}\right)}
\end{aligned}
$$

and

$$
\int_{\mathbb{R}^{d+1}}\left|\chi_{E}-\varphi_{\epsilon}\right| d P \leq \int_{U \backslash C} d P<\epsilon
$$

Since $\int_{\mathbb{R}^{d+1}} \varphi_{\epsilon}(x) F(x) d x=\int_{\mathbb{R}^{d+1}} \varphi_{\epsilon} d P$ we conclude that

$$
\begin{aligned}
\left|\int_{E} F(x) d x-P(E)\right| \leq & \int_{\mathbb{R}^{d+1}}\left|\chi_{E}-\varphi_{\epsilon}\right||F(x)| d x+\int_{\mathbb{R}^{d+1}}\left|\chi_{E}-\varphi_{\epsilon}\right| d P \\
& \leq \epsilon+\sqrt[q^{*}]{\epsilon} \cdot\|F\|_{L^{q}\left(\mathbb{R}^{d+1}\right)}
\end{aligned}
$$


and since $\epsilon$ is arbitrary we find that for any bounded Borel set $E, P(E)=\int_{E} F(x) d x$. Next, given any Borel set $E$, define $E_{m}=E \cap B_{m}(0)$. We have that $E_{m} \uparrow E$ and as such $P(E)=\lim _{m \rightarrow \infty} P\left(E_{m}\right)$. Using Fatu's lemma we find that

$$
\int_{E} F(x) d x=\int_{\mathbb{R}^{d}} \lim _{m \rightarrow \infty} \chi_{E_{m}} F(x) d x \leq \liminf _{m \rightarrow \infty} \int_{E_{m}} F(x) d x=\liminf _{m \rightarrow \infty} P\left(E_{m}\right)=P(E)
$$

If we'll prove that $F \in L^{1}\left(\mathbb{R}^{d+1}\right)$ we would be able to use the Dominated Convergence Theorem to show equality in the above inequality and conclude that $d P=F(x) d x$.

Since $F$ is continuous, if $\operatorname{Im} F\left(x_{0}\right) \neq 0$ for one point, we can find a ball around it, $B_{r}\left(x_{0}\right)$ such that $\operatorname{Im} F \neq 0$ in the entire ball. Since any ball is a bounded Borel set we have that

$$
P\left(B_{r}\left(x_{0}\right)\right)=\int_{B_{r}\left(x_{0}\right)} F(x) d x \notin \mathbb{R}
$$

which is impossible. Thus $F$ is real valued.

A similar argument shows that $F$ is positive. Indeed, if $F\left(x_{0}\right)<0$ for one point we can find a ball around it, $B_{r}\left(x_{0}\right)$ such that $F<0$ in that ball. We have that

$$
0>\int_{B_{r}\left(x_{0}\right)} F(x) d x=P\left(B_{r}\left(x_{0}\right)\right)
$$

again, impossible.

Thus $F \geq 0$ and we have that

$$
\int_{\mathbb{R}^{d+1}}|F(x)| d x=\int_{\mathbb{R}^{d+1}} F(x) d x \leq P\left(\mathbb{R}^{d+1}\right)=1
$$

completing our proof.

The last two Lemmas provide the proof to Lemma 3.3.

Proof of Lemma 3.3. Due to Lemma A.2, $\widehat{h_{\delta}} \in L^{2}\left(\mathbb{R}^{d+1}\right) \cap L^{1}\left(\mathbb{R}^{d+1}\right)$ for all $n>$ $\frac{2(1+d)}{d}$. As such, it has an inverse Fourier transform $F_{n} \in L^{2}\left(\mathbb{R}^{d+1}\right) \cap C\left(\mathbb{R}^{d+1}\right)$. Given any $\varphi \in C_{c}\left(\mathbb{R}^{d+1}\right)$ we have that

$\int_{\mathbb{R}^{d+1}} \varphi(u, v) d h^{* n}(u, v)=\int_{\mathbb{R}^{d+1}} \widehat{\varphi}(p, t){\overline{{\widehat{h_{\delta}}}^{n}}}(p, t) d p d t=\int_{\mathbb{R}^{d+1}} \varphi(u, v) \overline{F_{n}}(u, v) d u d v$

By Lemma A.3 we conclude that $F_{n} \geq 0$ and that $d h(u, v)=F_{n}(u, v) d u d v$.

\section{REFERENCES}

[1] A. V. Bobylev and C. Cercignani, On the Rate of Entropy Production for the Boltzmann Equation, J. Statist. Phys., 94, (1999), 603-618.

[2] E. A. Carlen, M. C. Carvalho and M. Loss, Many Body Aspects of Approach to Equilibrium, “Séminaire Equations aux Dérivées Partielles" (La Chapelle sur Erdre, 2000), Exp. No. XI, 12 pp., Univ. Nantes, Nantes, 2000.

[3] E. A. Carlen, M. C. Carvalho, J. Le Roux, M. Loss and C. Villani, Entropy and Chaos in the Kac Model, Kinet. Relat. Models, 3 (2010), 85-122.

[4] E. A. Carlen, J. S. Geronimo, M. Loss, Determination of the spectral gap in the Kac model for physical momentum and energy-conserving collisions, SIAM J. Math. Anal. 40 (2008), no. 1, 327-364.

[5] K. Carrapatoso, Quantative and Qualitative Kac's Chaos on the Boltzmann Sphere, Preprint 
[6] E. Amit, On Villani’s Conjecture Concerning Entropy Production for the Kac Master Equation, Kinet. Relat. Models, 4 (2011), no. 2, 479-497.

[7] E. Janvresse, Spectral Gap for Kac's Model of Boltzmann Equation, Ann. Probab., 29 (2001), 288-304.

[8] M. Kac, Foundations of Kinetic Theory, Proceedings of the Third Berkeley Symposium on Mathematical Statistics and Probability, 1954-1955, vol. III, pp. 171-197. University of California Press, Berkeley and Los Angeles, 1956.

[9] D. K. Maslen, The Eigenvalues of Kac's Master Equation, Math. Z. 243 (2003), no. 2, 291-331.

[10] H. P. Jr. McKean, An Exponential Formula for Solving Boltmann's Equation for a Maxwellian Gas, J. Combinatorial Theory 21967 358-382.

[11] S. Mischlet, C. Mouhot, Kac's Program in Kinetic Theory, Preprint

[12] C. Villani, Cercignani's Conjecture is Sometimes True and Always Almost True, Comm. Math. Phys., 234 (2003), 455-490.

[13] C. Villani A review of mathematical topics in collisional kinetic theory, Handbook of mathematical fluid dynamics, Vol. I, 71-305, North-Holland, Amsterdam, 2002. 